\title{
Conditions to Provable System-Wide Optimal Coordination of Connected and Automated Vehicles ${ }^{1}$
}

\author{
A M Ishtiaque Mahbub ${ }^{a}$, Andreas A. Malikopoulos $^{\text {a }}$ \\ ${ }^{a}$ Department of Mechanical Engineering, University of Delaware, 126 Spencer Lab, 130 Academy St, Newark DE 19716
}

\begin{abstract}
Connected and automated vehicles (CAVs) provide the most intriguing opportunity to improve energy efficiency, traffic flow, and safety. In earlier work, we addressed the constrained optimal coordination problem of CAVs at different traffic scenarios using Hamiltonian analysis. In this paper, we investigate the properties of the unconstrained problem and provide conditions under which different combination of the state and control constraints become active. We present a condition-based computational framework that improves on the standard iterative solution procedure of the constrained Hamiltonian analysis. Finally, we derive a closed-form analytical solution of the constrained optimal control problem and validate the proposed framework using numerical simulation. The solution can be derived without any recursive steps, and thus it is appropriate for real-time implementation on-board the CAVs.
\end{abstract}

Key words: Connected and automated vehicles; decentralized optimal control; energy usage.

\section{Introduction}

\subsection{Motivation}

The implementation of an emerging transportation system with connected and automated vehicles (CAVs) enables a novel computational framework to provide realtime control actions that optimize energy consumption and associated benefits. From a control point of view, CAVs can alleviate congestion at different traffic scenarios, reduce emission, improve fuel efficiency and increase passenger safety; see Margiotta and Snyder (2011); Malikopoulos et al. (2018). Urban intersections, merging roadways, highway on-ramps, roundabouts and speed reduction zones along with the driver responses to various disturbances are the primary sources of bottlenecks that contribute to traffic congestion; see Malikopoulos and Aguilar (2013).

\subsection{Literature Review}

Several research efforts have used optimal control theory to investigate how CAVs can potentially improve energy efficiency and travel time in these traffic scenarios.

Email addresses: mahbub@udel.edu (A M Ishtiaque Mahbub), andreas@udel.edu (Andreas A. Malikopoulos).

1 This research was supported by ARPAE's NEXTCAR program under the award number DE-AR0000796.
Early efforts reported in Levine and Athans (1966) and Athans (1969) considered a single string of vehicles that was coordinated through a traffic conflict zone with a linear optimal regulator. Shladover et al. (1991) discussed the lateral and longitudinal control of CAVs for the automated platoon formation. Varaiya (1993) outlined the key features of an automated intelligent vehicle/highway system, and proposed a basic control system architecture. Dresner and Stone (2004) proposed the use of the reservation scheme to control a signal-free intersection of two roads. Since then, several research efforts have considered reservation approaches for coordination of CAVs at urban intersections; see Dresner and Stone (2008); de La Fortelle (2010); Huang et al. (2012); Au and Stone (2010). Alonso et al. (2011) proposed a control framework where a CAV can derive its safe crossing schedule to avoid collision with a human-driven vehicle. Several approaches for coordinating CAVs that have been reported in the literature have proposed the use of centralized control, where there is at least one task in the system that is globally decided for all vehicles by a single central controller; see Dresner and Stone (2008); de La Fortelle (2010); Huang et al. (2012); Lu and Hedrick (2003); Xu et al. (2018); Bakibillah et al. (2019). Some approaches have focused on coordinating CAVs at intersections to improve traffic flow; see Yan et al. (2009); Kim and Kumar (2014), or travel time; see Raravi et al. (2007), while other approaches have focused on energy consumption improvement; see Mahler and Vahidi (2014); Sciarretta 
et al. (2015); Wan et al. (2016).

Some optimal control approaches reported in the literature have used standard Hamiltonian analysis for CAV control and coordination, e.g., Zhao et al. (2019); Wang et al. (2019); while other approaches have employed model predictive control; see Kim and Kumar (2014); Makarem and Gillet (2012). Dynamic programming (DP) has also been used to compute the optimal control input for CAVs, e.g., Ozatay et al. (2017), Mahler and Vahidi (2014), and Pei et al. (2019). DP, however, may not be feasible for real-time implementation due to its high required computational effort. In optimal control approaches, the problem formulation may have different objective functions including vehicle travel time, e.g., Raravi et al. (2007), energy consumption, e.g., Sciarretta et al. (2015), passenger comfort, e.g., Ntousakis et al. (2016), etc. Raravi et al. (2007) formulated an optimization problem the solution of which aims at finding the minimum time once the merging sequence is determined. Kamal et al. (2013) proposed numerical algorithms based on Pontryagin's minimum principle for CAV coordination in a signal-free intersection. A virtual platoon-based cooperative control approach was discussed in Huang et al. (2019) for on-ramp coordination. A hierarchical control framework using an upper-level CAV coordination and a low-level multiobjective optimization scheme was proposed in Qian et al. (2015). A similar hierarchical control framework has been reported by Bakibillah et al. (2019), where a two-level combinatorial optimization problem is formulated for a cloud-based roundabout coordination system.

In optimal control approaches, one key challenge is to handle the associated state, control and safety constraints. Min et al. (2019) considered a platoon-based approach to coordinate CAVs through a merging roadway, and solved the constrained optimization problem with distributed model predictive control. Sciarretta et al. (2015) developed an eco-driving controller for CAVs for adaptive cruise control maneuver, where the optimal control problem minimizes the energy consumption with speed constraint. Wan et al. (2016) proposed a speed advisory system to minimize fuel consumption without considering the state and control constraints. Han et al. (2018) proposed a safety based eco-driving control for the CAVs. Wang et al. (2019) formulated the multi-objective optimization problem for the CAVs approaching intersection, and derived the analytic solution based on the Pontraygin's minimum principle. Ozatay et al. (2017) provided a speed profile optimization framework for minimizing fuel consumption without considering any safety or acceleration/deceleration constraints.

Recently, a decentralized optimal control framework was presented for coordinating CAVs in real time at different traffic scenarios such as on-ramp merging roadways, roundabouts, speed reduction zones and signal- free intersections; see Malikopoulos et al. (2018); Mahbub et al. (2020a); Malikopoulos et al. (2019); Mahbub et al. (2020c). This framework uses a hierarchical structure consisting of an upper-level vehicle coordination problem to minimize travel time, and a low-level optimal control problem to minimize the energy of individual CAVs. A complete, analytical solution of the low-level control problem that includes the rear-end safety constraint, where the safe distance is a function of speed, was discussed in Malikopoulos and Zhao (2019b); Malikopoulos et al. (2021). A problem formulation for the upper-level optimization in which there is no duality gap, implying that the optimal time trajectory for each CAV does not activate any of the state, control, and safety constraints of the low-level optimization was presented in Malikopoulos and Zhao (2019a); Malikopoulos et al. (2021).

Detailed discussions of the research efforts reported in the literature to date on coordination of CAVs can be found in recent survey papers; see Rios-Torres and Malikopoulos (2017); Guanetti et al. (2018).

\subsection{Objectives and Contributions of the Paper}

The standard methodology to solve the low-level optimal control problem; see Malikopoulos et al. (2018); is to employ Hamiltonian analysis with interior point state and/or control constraints. Namely, we first start with the unconstrained arc and derive the solution of the lowlevel optimal control problem. If the solution violates any of the state or control constraints, then the unconstrained arc is pieced together with the arc corresponding to the violated constraint. The two arcs yield a set of algebraic equations which are solved simultaneously using the boundary conditions and interior constraints between the arcs. If the resulting solution, which includes the determination of the optimal switching time from one arc to the next one, violates another constraint, then the last two arcs are pieced together with the arc corresponding to the new violated constraint, and we re-solve the problem with the three arcs pieced together. The three arcs will yield a new set of algebraic equations that need to be solved simultaneously using the boundary conditions and interior constraints between the arcs. The resulting solution includes the optimal switching time from one arc to the next one. The process is repeated until the solution does not violate any other constraints. This recursive process of piecing the arcs together to derive the optimal solution of the low-level problem can be computationally expensive and might prevent real-time implementation.

In this paper, we provide an in-depth analysis of different state and control constraint activation cases, and establish a rigorous framework that yields a closed-form analytical solution for the low-level optimal control problem formulation without requiring the recursive process de- 
scribed above. Thus, the proposed framework is appropriate for real-time implementation on-board the CAVs; see Mahbub et al. (2020b). The objectives of this paper are (i) to derive a priori the different state and control constraint activation cases through a rigorous mathematical analysis, (ii) to simplify the recursive process required to derive the optimal constrained solution of the Hamiltonian analysis for the low-level optimal control problem, and (iii) to increase the computational efficiency of the derivation of the solution in (i) by eliminating numerical computations.

Thus, the contributions of this paper are: (1) an in-depth exposition of the properties of the different combinations of the state and control constraint activation cases and a set of a priori conditions to identify the constrained solution without any recursive steps, and (2) an explicit expression of the junction point between the constrained and unconstrained arcs leading to a closed-form analytical solution of the constrained optimal control problem. In earlier work, we reported a limited-scope analysis along with some preliminary results about the conditions for state and control constraint activation; see Mahbub and Malikopoulos (2020).

\subsection{Comparison With Related Work}

The framework that we report in this paper advances the state of the art in the following ways. First, the solution to the state and control unconstrained control problem presented in Malikopoulos et al. (2019) and Ntousakis et al. (2016) shows acceleration spikes (jerk) at the boundaries of the optimization horizon, possibly exceeding the vehicle's physical limitation and giving rise to undesired driving experience. In addition, the unconstrained solution can only guarantee that none of the constraints are violated at the boundaries of the optimization horizon only. In our proposed framework, we can guarantee that none of the the state and control constraints are violated throughout the entire optimization horizon. Second, in contrast to some approaches reported in the literature, e.g., Wan et al. (2016), Ozatay et al. (2017) and Han et al. (2018), where either the state or the control constrained optimal control problem was addressed, our framework addresses all state and control constraints cases. Moreover, we explicitly include the state and control constraints in the Hamiltonian analysis as opposed to using a feasibility zone; see Wang et al. (2019). Third, several approaches have considered free terminal time to address the state/control constraints within the optimization horizon; see Wang et al. (2019); Zhang and Cassandras (2019). In contrast, in our framework, we incorporate the constraints in the low-level control problem with the fixed time horizon. Fourth, the solution of the constrained optimal control problem requires piecing the unconstrained and constrained arcs together resulting in recursive numerical computations until all of the constraint activation cases are resolved; see Malikopoulos et al. (2018), Malikopoulos and Zhao (2019b) and Zhang and Cassandras (2019). In our proposed framework, we eliminate this recursive procedure to derive a real-time implementable closed-form analytical solution. Finally, the solution of the constrained optimization problem using Hamiltonian analysis reported in some approaches, e.g., Malikopoulos et al. (2018), Malikopoulos and Zhao (2019b) and Zhang and Cassandras (2019), only addresses different constraint activation cases without addressing the explicit interdependence between multiple constraint activation. In this paper, we explore the interdependence of the combination of the constraint activation cases and explicitly provide the conditions for their realization.

\subsection{Organization of the paper}

The remainder of the paper is organized as follows. In Section II, we introduce the problem formulation and present the unconstrained case. In Section III, we discuss different aspects of the state and control constrained formulation in detail. In Section IV, we provide the closedform analytical solution of the constrained optimal control problem. In Section V, we evaluate the effectiveness of the proposed approach in a simulation environment. Finally, we draw concluding remarks and discuss potential directions for future research in Section VI.

\section{Problem Formulation}

We consider CAVs travelling through a traffic network containing a four-way signal-free intersection, as shown in Fig. 1. Although our analysis can be applied to any traffic scenario, e.g., merging at roadways, roundabouts, and passing through speed reduction zones, we use an intersection (Fig. 1) as a reference to present the fundamental ideas and results of this paper, since an intersection provides unique features making it technically more challenging compared to other traffic scenarios. We define the area illustrated by the red square of dimension $S$ in Fig. 1 as the merging zone where potential lateral collision of CAVs may occur. Upstream of the merging zone, we define a control zone of length $L$ inside of which CAVs can communicate with each other using a vehicleto-vehicle communication protocol; see Mahbub et al. (2020b). The intersection also has a coordinator that communicates with the CAVs traveling inside the control zone. Note that, the coordinator does not make any decisions for the CAVs. When a CAV enters the control zone, the coordinator receives its information and assigns a unique identity $i \in \mathbb{N}$ to it. Let $\mathcal{N}(t)=\{1, \ldots, N(t)\}$, where $N(t) \in \mathbb{N}$ is the number of CAVs inside the control zone at time $t \in \mathbb{R}^{+}$, be the queue of CAVs to enter the merging zone shown in Fig. 1. The time that a CAV $i \in \mathcal{N}(t)$ enters the control and merging zones is denoted by $t_{i}^{0}$ and $t_{i}^{m}$, respectively, while the time that a CAV $i$ exits the merging zone is denoted by $t_{i}^{f}$. In our exposition, we assume that the queue $\mathcal{N}(t)$ and the optimal 
time to enter the merging zone $t_{i}^{m}$ is given a priori and can be derived by solving an upper-level vehicle coordination problem subject to rear-end and lateral safety constraints, as detailed in Malikopoulos et al. (2018); Mahbub et al. (2019, 2020a). Given $t_{i}^{m}$ a priori, the objective of each CAV $i \in \mathcal{N}(t)$ is to derive its optimal control input (acceleration/deceleration) to cross the intersection without any lateral or rear-end collision with the other CAVs, and without violating any of the state and control constraints.

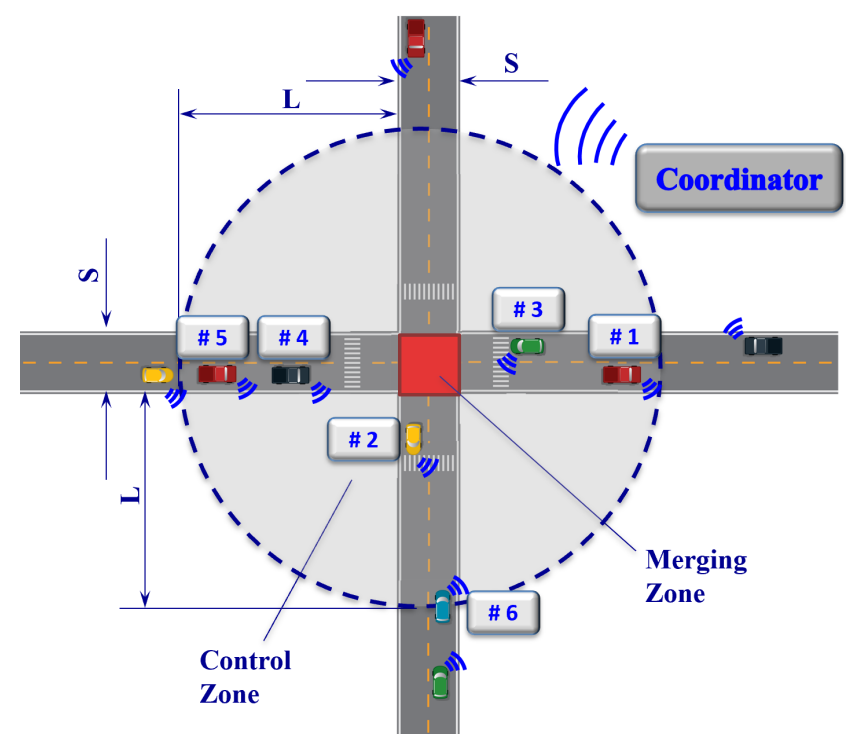

Fig. 1. A traffic network of connected automated vehicles approaching a four-way signal-free intersection.

\subsection{Modeling Framework}

We model each CAV $i \in \mathcal{N}(t)$ as a double integrator

$$
\dot{p}_{i}(t)=v_{i}(t), \dot{v}_{i}(t)=u_{i}(t), t \in\left[t_{i}^{0}, t_{i}^{f}\right],
$$

where $p_{i}(t) \in \mathcal{P}_{i}, v_{i}(t) \in \mathcal{V}_{i}$, and $u_{i}(t) \in \mathcal{U}_{i}$ denote the position, speed and acceleration (control input) of each CAV $i \in \mathcal{N}(t)$. The sets $\mathcal{P}_{i}, \mathcal{V}_{i}$, and $\mathcal{U}_{i}, i \in \mathcal{N}(t)$, are complete and totally bounded subsets of $\mathbb{R}$. Let $\mathbf{x}_{i}(t)=$ $\left[p_{i}(t) v_{i}(t)\right]^{T}$ denote the state vector of each CAV $i$, with initial value $\mathbf{x}_{i}^{0}=\left[\begin{array}{ll}p_{i}^{0} & v_{i}^{0}\end{array}\right]^{T}$ taking values in $\mathcal{X}_{i}=\mathcal{P}_{i} \times \mathcal{V}_{i}$. The state space $\mathcal{X}_{i}$ for each CAV $i$ is closed with respect to the induced topology on $\mathcal{P}_{i} \times \mathcal{V}_{i}$ and thus, it is compact.

To ensure that the control input and speed of each CAV $i \in \mathcal{N}$ are within a given admissible range, we impose the following constraints

$$
\begin{gathered}
u_{i, \min } \leq u_{i}(t) \leq u_{i, \max }, \quad \text { and } \\
0 \leq v_{\min } \leq v_{i}(t) \leq v_{\max }, \quad t \in\left[t_{i}^{0}, t_{i}^{f}\right],
\end{gathered}
$$

where $u_{i, \min }, u_{i, \max }$ are the minimum and maximum acceleration for each CAV $i \in \mathcal{N}(t)$, and $v_{\text {min }}, v_{\max }$ are the minimum and maximum speed limits respectively. Without loss of generality, we assume homogeneity in terms of CAV types, which enables the use of the same maximum acceleration $u_{\max }$ and minimum acceleration $u_{\text {min }}$ for any CAV $i \in \mathcal{N}(t)$. To ensure the avoidance of rear-end collision of two consecutive CAVs traveling on the same lane, we impose the rear-end safety constraint

$$
s_{i}(t) \geq \delta_{i}(t), \quad i \in \mathcal{N}(t), t \in\left[t_{i}^{0}, t_{i}^{f}\right]
$$

where $s_{i}(t):=p_{k}(t)-p_{i}(t)$ is defined as the distance between $\mathrm{CAV} i, k \in \mathcal{N}(t)$, where CAV $k$ is physically located immediately ahead of CAV $i$, and $\delta_{i}(t)$ is the minimum safe distance which is a function of speed $v_{i}(t)$. For each CAV $i \in \mathcal{N}(t)$, we define the set $\Gamma_{i}:=\{t \mid t \in$ $\left.\left[t_{i}^{m}, t_{i}^{f}\right]\right\}$. Lateral collision between any two CAVs $i, j \in$ $\mathcal{N}(t)$ can be avoided if

$$
\Gamma_{i} \cap \Gamma_{j}=\varnothing, \quad i, j \in \mathcal{N}(t), t \in\left[t_{i}^{m}, t_{i}^{f}\right]
$$

In the modeling framework described above, we impose the following assumptions:

Assumption 1 Each $C A V i \in \mathcal{N}(t)$ communicates with each other and with the coordinator without any delays or errors.

Assumption 2 For each $C A V i \in \mathcal{N}(t)$, no lane change maneuver is allowed within the control zone.

Assumption 3 None of the state constraints are active at time $t_{i}^{0}$ when each $C A V i \in \mathcal{N}(t)$ enters the control zone.

The first assumption may be strong but it is relatively straightforward to relax it as long as the noise in the measurements and/or delays is bounded. For example, we can determine upper bounds on the state uncertainties as a result of sensing or communication errors and delays, and incorporate these into more conservative safety constraints. The second assumption allows us to focus only on the control of longitudinal vehicle dynamics of CAVs within the control zone. Each CAV $i \in \mathcal{N}(t)$, however, can change lanes before the entry and/or after the exit of the control zone. Our analysis can include multiple lanes by appropriately revising the vehicle dynamics model (1). Finally, the third assumption ensures that, for each CAV $i \in \mathcal{N}(t)$, the initial state at the entry of the control zone is feasible.

\subsection{Low-level Optimal Control Problem}

For each CAV $i \in \mathcal{N}(t), t \in\left[t_{i}^{0}, t_{i}^{m}\right]$, traveling inside the control zone, we formulate the following optimal control 
problem

$$
\begin{gathered}
\min _{u_{i}(t) \in U_{i}} \int_{t_{i}^{0}}^{t_{i}^{m}} \frac{1}{2} u_{i}^{2}(t) d t, \\
\text { subject to : }(1),(2), p_{i}\left(t_{i}^{0}\right)=0, p_{i}\left(t_{i}^{m}\right)=L, \\
\text { and given } t_{i}^{0}, v_{i}\left(t_{i}^{0}\right), t_{i}^{m},
\end{gathered}
$$

where we consider the $L^{2}$-norm of the control input, i.e., $u_{i}^{2}(t)$, as the cost function. By minimizing transient engine operation, we have direct benefits in fuel consumption in conventional vehicles (vehicles with internal combustion engines); see Malikopoulos et al. (2018). Note that we do not explicitly include the lateral (4) and rearend (3) safety constraints in (5). The lateral collision constraint is enforced by selecting the appropriate merging time $t_{i}^{m}$ for each CAV $i$ in the upper-level throughput maximization problem. The activation of rear-end safety constraint can be avoided under certain conditions; see Malikopoulos et al. (2019).

In our formulation, the state constraints are $\mathbf{S}_{i}\left(t, \mathbf{x}_{i}(t)\right):=$ $\left[v_{i}(t)-v_{\max } v_{\text {min }}-v_{i}(t)\right]^{T} \leq 0$. Note that, $\mathbf{S}_{i}\left(t, \mathbf{x}_{i}(t)\right)$ is not an explicit function of the control input $u_{i}(t)$. Thus, to formulate the tangency constraints, we need to take successive time derivatives of $\mathbf{S}_{i}\left(t, \mathbf{x}_{i}(t)\right)$ until we obtain an expression that is explicitly dependent on $u_{i}(t)$; see Bryson and Ho (1975). If $q$ time derivatives are required, we refer to each constraint in $\mathbf{S}_{i}^{(q)}\left(t, \mathbf{x}_{i}(t)\right)$ as the $q$ th-order state variable inequality constraint. In our case, we have 1st-order speed constraint, e.g., $\mathbf{S}_{i}^{(1)}\left(t, \mathbf{x}_{i}(t), u_{i}(t)\right)=\left[\begin{array}{r}u_{i}(t) \\ -u_{i}(t)\end{array}\right]$.

To derive an analytical solution of the optimal control problem in (5) for each CAV $i \in \mathcal{N}(t)$, we formulate the adjoined Hamiltonian function $H_{i}\left(t, \mathbf{x}_{i}(t), u_{i}(t)\right), \quad t \in$ $\left[t_{i}^{0}, t_{i}^{m}\right]$, as follows,

$$
\begin{aligned}
& H_{i}\left(t, \mathbf{x}_{i}(t), u_{i}(t)\right)=\frac{1}{2} u_{i}^{2}(t)+\lambda_{i}^{p}(t) \cdot v_{i}(t)+\lambda_{i}^{v}(t) \cdot u_{i}(t) \\
& +\boldsymbol{\mu}_{i}^{T}(t) \cdot \mathbf{C}_{i}\left(t, \mathbf{x}_{i}(t), u_{i}(t)\right)+\boldsymbol{\eta}_{i}^{T}(t) \cdot \mathbf{S}_{i}\left(t, \mathbf{x}_{i}(t)\right) \\
& =\frac{1}{2} u_{i}^{2}(t)+\lambda_{i}^{p}(t) \cdot v_{i}(t)+\lambda_{i}^{v}(t) \cdot u_{i}(t) \\
& +\mu_{i}^{a}(t) \cdot\left(u_{i}(t)-u_{\max }\right)+\mu_{i}^{b}(t) \cdot\left(u_{\min }-u_{i}(t)\right) \\
& +\eta_{i}^{c}(t) \cdot\left(v_{i}(t)-v_{\max }\right)+\eta_{i}^{d}(t) \cdot\left(v_{\min }-v_{i}(t)\right),
\end{aligned}
$$

where, $\mathbf{C}_{i}\left(t, \mathbf{x}_{i}(t), u_{i}(t)\right):=\left[u_{i}(t)-u_{\max } u_{\min }-u_{i}(t)\right]^{T}$ is the vector of control constraints in $(2), \lambda_{i}^{p}(t), \lambda_{i}^{v}(t)$ are the co-state components corresponding to the state vector $\mathbf{x}_{i}(t)$, and $\boldsymbol{\mu}_{i}(t)$ is the path co-vector for control constraints consisting of the Lagrange multipliers with the following conditions,

$$
\begin{aligned}
& \mu_{i}^{a}(t)=\left\{\begin{array}{l}
>0, u_{i}(t)-u_{\max }=0, \\
=0, u_{i}(t)-u_{\max }<0,
\end{array}\right. \\
& \mu_{i}^{b}(t)=\left\{\begin{array}{l}
>0, u_{\min }-u_{i}(t)=0, \\
=0, u_{\min }-u_{i}(t)<0,
\end{array}\right.
\end{aligned}
$$

and $\boldsymbol{\eta}_{i}(t)$ is the path co-vector for state constraints consisting of the Lagrange multipliers,

$$
\begin{aligned}
& \eta_{i}^{c}(t)=\left\{\begin{array}{l}
>0, v_{i}(t)-v_{\max }=0, \\
=0, v_{i}(t)-v_{\text {max }}<0,
\end{array}\right. \\
& \eta_{i}^{d}(t)=\left\{\begin{array}{l}
>0, v_{\text {min }}-v_{i}(t)=0, \\
=0, v_{\text {min }}-v_{i}(t)<0 .
\end{array}\right.
\end{aligned}
$$

The corresponding Euler-Lagrange equations at time $t \in$ $\left[t_{i}^{0}, t_{i}^{m}\right]$ are

$$
\begin{gathered}
\dot{\lambda}_{i}^{p}(t)=-\frac{\partial H_{i}}{\partial p_{i}}=0, \\
\dot{\lambda}_{i}^{v}(t)=-\frac{\partial H_{i}}{\partial v_{i}}= \begin{cases}-\lambda_{i}^{p}(t), & v_{i}(t)-v_{\max }<0 \\
-\lambda_{i}^{p}(t)-\eta_{i}^{c}(t), & v_{i}(t)-v_{\max }=0, \\
-\lambda_{i}^{p}(t)+\eta_{i}^{d}(t), & v_{\min }-v_{i}(t)=0,\end{cases}
\end{gathered}
$$

and

$$
\frac{\partial H_{i}}{\partial u_{i}}=u_{i}(t)+\lambda_{i}^{v}(t)+\mu_{i}^{a}(t)-\mu_{i}^{b}(t)=0 .
$$

If the inequality state and control constraints (2) are not active, we have $\mu_{i}^{a}(t)=\mu_{i}^{b}(t)=\eta_{i}^{c}(t)=\eta_{i}^{d}(t)=0$. Applying the necessary conditions, the optimal control $u_{i}^{*}(t)$ can be derived from $u_{i}^{*}(t)+\lambda_{i}^{v}(t)=0, i \in \mathcal{N}(t)$. From (11) and (12) we have $\lambda_{i}^{p}(t)=a_{i}$, and $\lambda_{i}^{v}(t)=$ $-\left(a_{i} \cdot t+b_{i}\right)$, where $a_{i}$ and $b_{i}$ are constants of integration corresponding to each CAV $i \in \mathcal{N}(t)$. Therefore, the unconstrained optimal control input $u_{i}^{*}(t)$ is

$$
u_{i}^{*}(t)=a_{i} \cdot t+b_{i}, t \in\left[t_{i}^{0}, t_{i}^{m}\right] .
$$

Substituting the last equation into (1) we find the optimal speed and position for each CAV $i \in \mathcal{N}(t)$, namely

$$
\begin{aligned}
& v_{i}^{*}(t)=\frac{1}{2} a_{i} \cdot t^{2}+b_{i} \cdot t+c_{i}, \\
& p_{i}^{*}(t)=\frac{1}{6} a_{i} \cdot t^{3}+\frac{1}{2} b_{i} \cdot t^{2}+c_{i} \cdot t+d_{i}, t \in\left[t_{i}^{0}, t_{i}^{m}\right],
\end{aligned}
$$

where $c_{i}$ and $d_{i}$ are constants of integration corresponding to each CAV $i \in \mathcal{N}(t)$. The constants of integration 
$a_{i}, b_{i}, c_{i}$, and $d_{i}$ can be determined from (14)-(16) using the initial and boundary conditions imposed in (5). Note that, we can either compute $a_{i}, b_{i}, c_{i}$, and $d_{i}$ only once at time $t=t_{i}^{0}$ and apply the solution throughout optimization horizon $\left[t_{i}^{0}, t_{i}^{m}\right]$, or update the constants of integration by recomputing (14)-(16) at some discrete time step in $\left[t_{i}^{0}, t_{i}^{m}\right]$ to account for any disturbance within the control zone. For the remainder of the paper, we reserve the notations $a_{i}, b_{i}, c_{i}$, and $d_{i}$ only for the unconstrained optimal solution given in (14)-(16).

Remark 1 For the case where the constants of integration $a_{i}=0$ and $b_{i}=0$, we have the trivial solution of the unconstrained problem (14)-(16) as $u_{i}^{*}(t)=0, v_{i}^{*}(t)=$ $c_{i}, p_{i}^{*}(t)=c_{i} \cdot t+d_{i}, t \in\left[t_{i}^{0}, t_{i}^{m}\right]$. This implies that if the speed is constant and the speed constraint is not active at time $t=t_{i}^{0}$ (Assumption 3), none of the state and control constraints becomes active for $t \in\left[t_{i}^{0}, t_{i}^{m}\right]$. If $a_{i}, b_{i} \neq 0$, we have $u_{i}^{*}\left(t_{i}^{0}\right) \neq 0$.

In what follows, we only consider the non-trivial case (Remark 1) of the constrained optimization problem (5) where $a_{i}, b_{i} \neq 0$.

\section{Analysis of the Constrained Optimal Control Problem}

To derive the constrained analytical solution of (5), we follow the standard methodology used in optimal control problems with interior point state and/or control constraints; see Bryson et al. (1963); Bryson and Ho (1975). Namely, we first start with the unconstrained arc and derive the solution using (14)-(16). If the solution violates any of the state or control constraints, then the unconstrained arc is pieced together with the arc corresponding to the activated constraint, and we re-solve the problem with the two arcs pieced together at the junction point between the constrained and unconstrained arcs of the constrained solution (5). The two arcs yield a set of algebraic equations which are solved simultaneously using the boundary conditions of (5) and the interior conditions between the arcs. If the resulting solution, which includes the determination of the junction point from one arc to the next one, violates another constraint, then the last two arcs are pieced together with the arc corresponding to the new activated constraint, and we re-solve the problem with the three arcs pieced together. The three arcs will yield a new set of algebraic equations that need to be solved simultaneously using the boundary conditions of (5) and interior conditions between the arcs. The resulting solution includes the junction point from one arc to the next one. The process is repeated until the solution does not violate any other constraints.

This process can be computationally intensive for the following reasons. First, the recursive solution process to resolve all possible combinations of constraint activation might lead to intensive computation that prohibits real-time implementation. Second, each of the aforementioned recursion needs to be solved numerically due to the presence of implicit functions. To address both issues, we introduce a condition-based framework for the optimal control problem in (5) which leads to a closed-form analytical solution without this recursive procedure.

\subsection{Condition of Constraint Exclusion}

For the optimal control problem in (5), we have two state and two control constraints leading to 15 possible constraint combinations in total that can become active within the optimization horizon $\left[t_{i}^{0}, t_{i}^{m}\right]$. In this section, we show that it is only possible for a subset of the constraints to become active in $\left[t_{i}^{0}, t_{i}^{m}\right]$. Therefore, it is not necessary to consider all the cases in (5). In what follows, we delve deeper into the nature of the unconstrained optimal solution given in (14)-(16) to derive useful information about the possible existence of constraint activation within the control zone.

Lemma 1 For each $C A V i \in \mathcal{N}(t)$, let $a_{i}$ and $b_{i}$ be the constants of integration of the unconstrained solution of (5) corresponding to the optimal control input $u_{i}^{*}(t), t \in$ $\left[t_{i}^{0}, t_{i}^{m}\right]$. If the speed $v_{i}(t)$ is not specified at $t_{i}^{m}$, then

$$
a_{i} \cdot t_{i}^{m}+b_{i}=0, \quad t_{i}^{m}>t_{i}^{0} \geq 0 .
$$

PROOF. For all $i \in \mathcal{N}(t)$, since the speed $v_{i}(t)$ at $t=$ $t_{i}^{m}>t_{i}^{0}$ is not fixed, we have $\lambda_{i}^{v}\left(t_{i}^{m}\right)=0$ (Naidu, 2002), which implies $u_{i}^{*}\left(t_{i}^{m}\right)=0$, and the result follows.

Corollary 1 The constants of integration $a_{i}$ and $b_{i}$ of the unconstrained solution of (5) have opposite signs.

PROOF. Since $t_{i}^{m}$ is positive and non-zero, the result follows from (17).

Corollary 2 The unconstrained optimal control input $u_{i}^{*}(t)$ is linearly either increasing or decreasing with respect to time, and $u_{i}^{*}\left(t_{i}^{m}\right)=0$.

PROOF. From $(14), u_{i}^{*}(t)$ is a linear function with $u_{i}^{*}\left(t_{i}^{0}\right) \neq 0$ for the non-trivial case (Remark 1), and $u_{i}^{*}\left(t_{i}^{m}\right)=0$ (Lemma 1), so the result follows.

Remark 2 The constants of integration $a_{i}$ and $b_{i}$ of the unconstrained solution of (5) represents the slope of $u_{i}^{*}(t), t \in\left[t_{i}^{0}, t_{i}^{m}\right]$, and the initial value of the control input $u_{i}^{*}(t)$ at time $t=t_{i}^{0}$, respectively.

Lemma 2 Let $_{i}\left(t_{i}^{0}\right)$ be the initial speed of $C A V i \in \mathcal{N}(t)$ when it enters the control zone at $p_{i}\left(t_{i}^{0}\right)$ and travels up to the entry of the merging zone at $p_{i}\left(t_{i}^{m}\right)$. Then the nature of the unconstrained optimal control input $u_{i}^{*}(t)$ can 
be characterized using the following conditions based on the boundary conditions of $v_{i}\left(t_{i}^{0}\right), p_{i}\left(t_{i}^{0}\right)$ and $p_{i}\left(t_{i}^{m}\right)$ : $(i)$ The unconstrained optimal control input $u_{i}^{*}(t)$ is linearly decreasing if $v_{i}\left(t_{i}^{0}\right)<\frac{\left(p_{i}\left(t_{i}^{m}\right)-p_{i}\left(t_{i}^{0}\right)\right)}{t_{i}^{m}}$. (ii) The unconstrained optimal control input $u_{i}^{*}(t)$ is linearly increasing if $v_{i}\left(t_{i}^{0}\right)>\frac{\left(p_{i}\left(t_{i}^{m}\right)-p_{i}\left(t_{i}^{0}\right)\right)}{t_{i}^{m}}$.

PROOF. From (15) and (16), we can write $v_{i}\left(t_{i}^{0}\right)=$ $\frac{1}{2} a_{i} \cdot\left(t_{i}^{0}\right)^{2}+b_{i} \cdot t_{i}^{0}+c_{i}$ and $p_{i}\left(t_{i}^{0}\right)=\frac{1}{6} a_{i} \cdot\left(t_{i}^{0}\right)^{3}+\frac{1}{2} b_{i}$. $\left(t_{i}^{0}\right)^{2}+c_{i} \cdot t_{i}^{0}+d_{i}$. Without loss of generality, if we let $t_{i}^{0}=0$, we have

$$
c_{i}=v_{i}\left(t_{i}^{0}\right), d_{i}=p_{i}\left(t_{i}^{0}\right)
$$

Evaluating (16) at $t=t_{i}^{m}$, we have $p_{i}\left(t_{i}^{m}\right)=\frac{1}{6} a_{i} \cdot\left(t_{i}^{m}\right)^{3}+$ $\frac{1}{2} b_{i} \cdot\left(t_{i}^{m}\right)^{2}+c_{i} \cdot t_{i}^{m}+d_{i}$. Substituting (17) and (18) in the above equation and solving for $a_{i}$, we have

$$
a_{i}=\frac{3\left(v_{i}\left(t_{i}^{0}\right) \cdot t_{i}^{m}-\left(p_{i}\left(t_{i}^{m}\right)-p_{i}\left(t_{i}^{0}\right)\right)\right)}{\left(t_{i}^{m}\right)^{3}}
$$

Since $t_{i}^{m}>0$, we have a non-positive constant of integration $a_{i}$, if $\left(v_{i}\left(t_{i}^{0}\right) \cdot t_{i}^{m}-\left(p_{i}\left(t_{i}^{m}\right)-p_{i}\left(t_{i}^{0}\right)\right)\right)<0$. From Corollary 2 and Remark 2 , a non-positive $a_{i}$ indicates a negative slope for $u_{i}^{*}(t)$, which implies that $u_{i}^{*}(t)$ is a linearly decreasing acceleration, and the proof is complete. The second part of Lemma 2 can be proved following similar steps, hence it is omitted.

Remark 3 When the $C A V i \in \mathcal{N}(t)$ travels with its initial speed $v_{i}\left(t_{i}^{0}\right)$ throughout the control zone, we have $v_{i}\left(t_{i}^{0}\right) \cdot t_{i}^{m}=\left(p_{i}\left(t_{i}^{m}\right)-p_{i}\left(t_{i}^{0}\right)\right)$. From (19), this implies that $a_{i}=0$, referring to an optimal control input $u_{i}^{*}(t)$ with horizontal slope. Since $u_{i}^{*}\left(t_{i}^{m}\right)=0$ (Lemma 1), we have $u_{i}^{*}(t)=0$, for all $t \in\left[t_{i}^{0}, t_{i}^{m}\right]$.

Lemma 3 For the unconstrained optimal solution of $(5)$, if either $v_{i}(t)-v_{\max } \leq 0$ or $u_{i}(t)-u_{\max } \leq 0$ becomes active at any time $t \in\left[t_{i}^{0}, t_{i}^{m}\right]$, neither $v_{\text {min }}-v_{i}(t) \leq 0$ nor $u_{\text {min }}-u_{i}(t) \leq 0$ can become active in $\left[t_{i}^{0}, t_{i}^{m}\right]$. The reverse also holds.

PROOF. Let $u_{i}^{*}(t)=a_{i} \cdot t+b_{i}>0>u_{\text {min }}$ at some time $t \in\left[t_{i}^{0}, t_{i}^{m}\right)$. Since $u_{i}^{*}\left(t_{i}^{m}\right)=0$ (Lemma 1) and $u_{i}^{*}(t)$ is a linearly decreasing function (Corollary 2), we have $u_{i}^{*}(t)>u_{\text {min }}$, for all $t \in\left[t_{i}^{0}, t_{i}^{m}\right]$, i.e., the constraint $u_{\text {min }}-u_{i}(t) \leq 0$ can not become active at any time in $t \in\left[t_{i}^{0}, t_{i}^{m}\right]$. The corresponding quadratic optimal speed profile $v_{i}^{*}(t)$ in (15) is a parabolic function of degree 2 with y-symmetric axis located at $t_{i}^{m}$ in the speed-time graph. Applying the necessary and sufficient condition of optimality in (15), we have

$$
\dot{v}_{i}^{*}(t)=a_{i} \cdot t+b_{i}=0, \ddot{v}_{i}^{*}(t)=a_{i}, t \in\left[t_{i}^{0}, t_{i}^{m}\right]
$$

Solving the first equation of (20), we have the extremum point at $t=-\frac{b_{i}}{a_{i}}$ which corresponds to the vertex of the parabola of (15) at $t=t_{i}^{m}$. Whether this point corresponds to the maximum or minimum of the (15) can be determined from the second part of $(20)$. Since $u_{i}^{*}(t)$ is decreasing, $a_{i}<0$ (Remark 2). Thus, the second equation of (20) indicates a maximum value at the vertex $t_{i}^{m}$, indicating a concave quadratic profile of $v_{i}^{*}(t)$. Since the extremum of the quadratic profile of $v_{i}^{*}(t)$ is located at $t_{i}^{m}$ and $v_{\min }<v_{i}\left(t_{i}^{0}\right)<v_{\max }$ (Assumption 3 ), we have $v_{i}^{*}(t)>v_{\text {min }}$ for all $t \in\left[t_{i}^{0}, t_{i}^{m}\right]$. Therefore, the constraints $v_{\min }-v_{i}(t) \leq 0$ can not become active at any time $t \in\left[t_{i}^{0}, t_{i}^{m}\right]$, and the proof of the first part of Lemma 3 is complete.

Conversely, let $u_{i}^{*}(t)=a_{i} \cdot t+b_{i}<0<u_{\max }$ at some $t \in$ $\left[t_{i}^{0}, t_{i}^{m}\right]$. Since $u_{i}^{*}\left(t_{i}^{m}\right)=0$ (Lemma 1$)$ and $u_{i}^{*}(t)$ is linearly increasing in $t \in\left[t_{i}^{0}, t_{i}^{m}\right]$ (Remark 2$), u_{\text {min }}-u_{i}(t) \leq 0$ can not become active at any $t \in\left[t_{i}^{0}, t_{i}^{m}\right]$. In addition, $u_{i}^{*}(t)$ yields a convex quadratic profile of $v_{i}^{*}(t)$ with vertex at $t=t_{i}^{m}$. Since the extremum point is located at $t_{i}^{m}$ and $v_{\min }<v_{i}\left(t_{i}^{0}\right)<v_{\max }$ (Assumption 3 ), we have $v_{i}^{*}(t)<v_{\max }$ for any $t \in\left[t_{i}^{0}, t_{i}^{m}\right]$, which implies that the state constraint $v_{i}(t)-v_{\max } \leq 0$ cannot become active at any time $t \in\left[t_{i}^{0}, t_{i}^{m}\right]$.

Corollary 3 The sign of $a_{i}$ corresponding to the unconstrained solution of (5) dictates the activation of either constraint set $\left\{v_{i}(t)-v_{\max } \leq 0, u_{i}(t)-u_{\max } \leq 0\right\}$ or $\left\{v_{\text {min }}-v_{i}(t) \leq 0, u_{\text {min }}-u_{i}(t) \leq 0\right\}$.

PROOF. Since $a_{i}$ is the slope of the optimal control input $u_{i}^{*}(t)$ (Remark 2), the sign of $a_{i}$ determines whether $u_{i}^{*}(t)$ is positive and decreasing or negative and increasing, which, in turn, determines the constraint activation criteria in Lemma 3.

Remark 4 The sign of $a_{i}$ can provide direct insight on which of the state and control constraints becomes active, and thus it can reduce the cardinality of the set of possible constrain activation cases.

Based on Lemmas 2 and 3, we now present the following result which provides the condition under which the state and control constraints become active. Note that the result is based on the initial and final conditions of (5) which enable the determination of the possible constraint activation set without solving the unconstrained optimization problem in (5).

Theorem 1 Let $C A V i \in \mathcal{N}(t)$ enter the control zone with initial speed $v_{i}\left(t_{i}^{0}\right)$ and travel with the unconstrained optimal control input $u_{i}^{*}(t), t \in\left[t_{i}^{0}, t_{i}^{m}\right]$. Then, (i) $v_{\text {min }}-$ $v_{i}(t) \leq 0$ and $u_{\text {min }}-u_{i}(t) \leq 0$ do not become active in $t \in\left[t_{i}^{0}, t_{i}^{m}\right]$, if $v_{i}\left(t_{i}^{0}\right)<\frac{\left(p_{i}\left(t_{i}^{m}\right)-p_{i}\left(t_{i}^{0}\right)\right)}{t_{i}^{m}}$, and (ii) $v_{i}(t)-$ $v_{\max } \leq 0$ and $u_{i}(t)-u_{\max } \leq 0$ do not become active in $t \in\left[t_{i}^{0}, t_{i}^{m}\right]$, if $v_{i}\left(t_{i}^{0}\right)>\frac{\left(p_{i}\left(t_{i}^{m}\right)-p_{i}\left(t_{i}^{0}\right)\right)}{t_{i}^{m}}$. 
PROOF. If $v_{i}\left(t_{i}^{0}\right)<\frac{\left(p_{i}\left(t_{i}^{m}\right)-p_{i}\left(t_{i}^{0}\right)\right)}{t_{i}^{m}}$, then from (19) $a_{i}<$ 0 , hence $u_{i}^{*}(t)$ is linearly decreasing (Lemma 2 ). Therefore, from Lemma $3, v_{\text {min }}-v_{i}(t) \leq 0$ and $u_{\text {min }}-u_{i}(t) \leq 0$ can not become active in $t \in\left[\overline{t_{i}^{0}}, t_{i}^{m}\right]$, which concludes the proof of the first part.

For the second part of Theorem 1, suppose that $v_{i}\left(t_{i}^{0}\right)>$ $\frac{\left(p_{i}\left(t_{i}^{m}\right)-p_{i}\left(t_{i}^{0}\right)\right)}{t_{i}^{m}}$. Hence $a_{i}>0$ (Lemma 2), and $u_{i}^{*}(t)$ is linearly increasing. Therefore, from Lemma $3, v_{i}(t)-$ $v_{\max }(t) \leq 0$ and $u_{i}(t)-u_{\max } \leq 0$ can not become active in $t \in\left[t_{i}^{0}, t_{i}^{m}\right]$, and the proof is complete.

Remark 5 Theorem 1 aims at reducing the possible set of constraint activation cases. For example, if the condition in part (i) of Theorem 1 holds, then from the 15 possible cases of constraint activation, we only need to consider 3 cases: $(a) v_{i}(t)-v_{\max } \leq 0$, (b) $u_{i}(t)-u_{\max } \leq 0$, and $(c)$ both $v_{i}(t)-v_{\max } \leq 0$ and $u_{i}(t)-u_{\max } \leq 0$. Similarly, if the condition in part (ii) of Theorem 1 holds, then from the 15 possible cases of constraint activation, we only need to consider 3 cases: (a) $v_{\text {min }}-v_{i}(t) \leq 0$, (b) $u_{\text {min }}-u_{i}(t) \leq 0$, and (c) both $v_{\min }-v_{i}(t) \leq 0$ and $u_{\text {min }}-u_{i}(t) \leq 0$.

Although Theorem 1 aims at reducing the possible constraint activation cases, it does not lead to the identification of the exact constraint activation of the unconstrained solution of (5). In what follows, we provide the conditions that can be used to extend the results of Theorem 1 and identify the activation of any constraint case in $\left[t_{i}^{0}, t_{i}^{m}\right]$.

\subsection{Conditions of Constraint Activation}

We start our exposition with some results that contain essential properties of the state and control constraint activation.

Lemma 4 If neither $u_{i}(t)-u_{\max } \leq 0$ nor $u_{\min }-u_{i}(t) \leq$ 0 is active at $t=t_{i}^{0}$, then it is guaranteed that neither of them will become active for all $t \in\left[t_{i}^{0}, t_{i}^{m}\right]$.

PROOF. Suppose that the unconstrained optimal solution of (5) yields $u_{i}^{*}(t)=a_{i} t+b_{i}$ with $a_{i}<0$. From Corollary 2 and Remark $2, u_{i}^{*}(t)$ decreases with respect to $t$, and at $t_{i}^{m}, u_{i}^{*}\left(t_{i}^{m}\right)=0$. Therefore, if $u_{i}^{*}\left(t_{i}^{0}\right)<u_{\text {max }}$, then $u_{i}^{*}(t)<u_{\max }$ for all $t \in\left[t_{i}^{0}, t_{i}^{m}\right]$. The second part of Lemma 4 can be proved following similar steps, hence it is omitted.

Lemma 5 If either $v_{i}(t)-v_{\max } \leq 0$ or $v_{\min }-v_{i}(t) \leq 0$ becomes active at any time $t \in\left[t_{i}^{0}, t_{i}^{m}\right)$, then it will remain active until $t=t_{i}^{m}$.

PROOF. Suppose that the unconstrained optimal solution of (5) yields $u_{i}^{*}(t)=a_{i} t+b_{i}$ with $a_{i}<0$. From
Corollary 2 and Remark $2, u_{i}^{*}(t)$ decreases with respect to $t$, and at $t_{i}^{m}, u_{i}^{*}\left(t_{i}^{m}\right)=0$, which implies that $v_{i}^{*}(t)$ is monotonically increasing, i.e., $v_{i}^{*}\left(t_{i}^{m}\right) \geq v_{i}^{*}(t)$ in $t \in$ $\left[t_{i}^{0}, t_{i}^{m}\right)$. Therefore, $v_{i}(t)-v_{\max } \leq 0$ will remain active until $t=t_{i}^{m}$. The second part of Lemma 5 can be proved following similar steps, hence it is omitted.

Remark 6 Lemma 4 implies that the entry of the control-constrained arc can be only at $t=t_{i}^{0}$, while Lemma 5 implies that there is no exit point in $\left[t_{i}^{0}, t_{i}^{m}\right]$ of the state-constrained arc after it becomes active.

The following results provide the conditions for which state and control constraint activation cases can be identified for the optimal control problem (5) a priori.

Theorem 2 Let $u_{i}^{*}(t)=a_{i} t+b_{i}, t \in\left[t_{i}^{0}, t_{i}^{m}\right]$, be the optimal control input of $C A V i \in \mathcal{N}(t)$ for the unconstrained solution of (5). Then, (i) for $a_{i}<0, v_{i}(t)-v_{\max } \leq 0$ becomes active if $t_{i}^{m} \leq \frac{3\left(p_{i}\left(t_{i}^{m}\right)-p_{i}\left(t_{i}^{0}\right)\right)}{v_{i}\left(t_{i}^{0}\right)+2 v_{\max }}$, and (ii) for $a_{i}>0$, $v_{\text {min }}-v_{i}(t) \leq 0$ becomes active if $t_{i}^{m} \geq \frac{3\left(p_{i}\left(t_{i}^{m}\right)-p_{i}\left(t_{i}^{0}\right)\right)}{v_{i}\left(t_{i}^{0}\right)+2 v_{\text {min }}}$.

PROOF. For $a_{i}<0$, suppose that there exists a time $t_{i}^{s} \in\left(t_{i}^{0}, t_{i}^{m}\right]$ at which $v_{i}(t)-v_{\max } \leq 0$ becomes active. Then, from (15) and (18), we have $\frac{1}{2} a_{i} \cdot\left(t_{i}^{s}\right)^{2}+$ $b_{i} \cdot t_{i}^{s}+v_{i}\left(t_{i}^{0}\right)=v_{\max }$. Solving the quadratic equation for $t_{i}^{s}$, we have $t_{i}^{s}=\frac{-2 b_{i} \pm \sqrt{4 b_{i}^{2}-8 a_{i} \cdot\left(v_{i}\left(t_{i}^{0}\right)-v_{\max }\right)}}{2 a_{i}}$, which yields $t_{i}^{s}=t_{i}^{m} \pm \sqrt{\frac{4 b_{i}^{2}-8 a_{i} \cdot\left(v_{i}\left(t_{i}^{0}\right)-v_{\max }\right)}{4 a_{i}^{2}}}$. Since $t_{i}^{s} \leq t_{i}^{m}$, a feasible solution of $t_{i}^{s}$ exists if we have $\sqrt{4 b_{i}^{2}-8 a_{i} \cdot\left(v_{i}\left(t_{i}^{0}\right)-v_{\max }\right)} \geq 0$ resulting in $a_{i} \leq$ $\frac{2\left(v_{i}\left(t_{i}^{0}\right)-v_{\max }\right)}{\left(t_{i}^{m}\right)^{2}}$. Combining with (19), the proof of the first statement of Theorem 2 follows.

For $a_{i}>0$, suppose that there exists a time $t_{i}^{s} \in\left(t_{i}^{0}, t_{i}^{m}\right]$ at which the state constraint $v_{\text {min }}-v_{i}(t) \leq 0$ becomes active. Then, from (15) and (18), we have $\frac{1}{2} a_{i} \cdot\left(t_{i}^{s}\right)^{2}+$ $b_{i} \cdot t_{i}^{s}+v_{i}\left(t_{i}^{0}\right)=v_{\min }$. Solving the above equation for $t_{i}^{s}$, we have $t_{i}^{s}=\frac{-2 b_{i} \pm \sqrt{4 b_{i}^{2}-8 a_{i} \cdot\left(v_{i}\left(t_{i}^{0}\right)-v_{\min }\right)}}{2 a_{i}}$, which yields $t_{i}^{s}=t_{i}^{m} \pm \sqrt{\frac{4 b_{i}^{2}-8 a_{i} \cdot\left(v_{i}\left(t_{i}^{0}\right)-v_{\min }\right)}{4 a_{i}^{2}}}$. Since $t_{i}^{s} \leq t_{i}^{m}$, we need to have $\sqrt{4 b_{i}^{2}-8 a_{i} \cdot\left(v_{i}\left(t_{i}^{0}\right)-v_{\text {min }}\right)} \geq 0$, and combining with (19), the proof of the second statement of Theorem 2 follows.

Theorem 3 Let $u_{i}^{*}(t)=a_{i} \cdot t+b_{i}, t \in\left[t_{i}^{0}, t_{i}^{m}\right]$, be the optimal control input of CAV $i \in \mathcal{N}(t)$ for the unconstrained solution of (5). Then, (i) for $a_{i}<0, u_{i}(t)-u_{\max } \leq 0$ becomes active if $t_{i}^{m} \leq$ $\frac{-3 v_{i}\left(t_{i}^{0}\right)+\sqrt{9\left(v_{i}\left(t_{i}^{0}\right)\right)^{2}+12 u_{\max } \cdot\left(p_{i}\left(t_{i}^{m}\right)-p_{i}\left(t_{i}^{0}\right)\right)}}{2 u_{\max }}, \quad$ and

for $a_{i}>0, u_{\min } u_{\max }-u_{i}(t) \leq 0$ becomes active if $t_{i}^{m} \geq \frac{-3 v_{i}\left(t_{i}^{0}\right)+\sqrt{9\left(v_{i}\left(t_{i}^{0}\right)\right)^{2}+12 u_{\min } \cdot\left(p_{i}\left(t_{i}^{m}\right)-p_{i}\left(t_{i}^{0}\right)\right)}}{2 u_{\min }}$. 
PROOF. For $a_{i}<0$, without loss of generality, we let $t_{i}^{0}=0$. Given $v_{i}\left(t_{i}^{0}\right), p_{i}\left(t_{i}^{0}\right)$ and $p_{i}\left(t_{i}^{m}\right)$, we will show that $t_{i}^{m}$ determines whether $u_{i}(t)-u_{\max } \leq 0$ becomes active or not. Let $\hat{t}_{i}^{m}$ be the value for which $u_{i}(t)-u_{\max } \leq 0$ becomes active at $t_{i}^{0}$, and $\hat{a}_{i}, \hat{b}_{i}$ the corresponding constants of integration. Then from (17) and (19), we can write $\hat{b}_{i}=$ $-\frac{3\left(v_{i}\left(t_{i}^{0}\right) \cdot \hat{t}_{i}^{m}-L\right)}{\left(\hat{t}_{i}^{m}\right)^{2}}=u_{\max }$, where $L=p_{i}\left(t_{i}^{m}\right)-p_{i}\left(t_{i}^{0}\right)=$ $p_{i}\left(\hat{t}_{i}^{m}\right)-p_{i}\left(t_{i}^{0}\right)$, which can be reduced to $u_{\max } \cdot\left(\hat{t}_{i}^{m}\right)^{2}+$ $3 v_{i}\left(t_{i}^{0}\right) \cdot \hat{t}_{i}^{m}-3 L=0$. The solution of the last equation yields $\hat{t}_{i}^{m}=\frac{-3 v_{i}\left(t_{i}^{0}\right) \pm \sqrt{\left.9\left(v_{i}\left(t_{i}^{0}\right)\right)^{2}+12 u_{\max } \cdot L\right)}}{2 u_{\max }}$. Since $\hat{t}_{i}^{m}>0$, $\hat{t}_{i}^{m}=\frac{-3 v_{i}\left(t_{i}^{0}\right)+\sqrt{\left.9\left(v_{i}\left(t_{i}^{0}\right)\right)^{2}+12 u_{\max } \cdot L\right)}}{2 u_{\max }}$. Hence, for any $t_{i}^{m}$ such that $t_{i}^{m} \leq \hat{t}_{i}^{m}, u_{i}(t)-u_{\max } \leq 0$ becomes active, and the proof of the first statement of Theorem 3 is complete.

For $a_{i}>0$, without loss of generality, we let $t_{i}^{0}=0$. Let $\hat{t}_{i}^{m}$ be a value that $u_{\text {min }}-u_{i}(t) \leq 0$ becomes active at $t_{i}^{0}$, and $\hat{a}_{i}, \hat{b}_{i}$ the corresponding constants of integration. Then from (17) and (19), we can write $\hat{b}_{i}=-\frac{3\left(v_{i}\left(t_{i}^{0}\right) \cdot \hat{t}_{i}^{m}-L\right)}{\left(\hat{t}_{i}^{m}\right)^{2}}=u_{\text {min }}$, where $L=$ $p_{i}\left(t_{i}^{m}\right)-p_{i}\left(t_{i}^{0}\right)=p_{i}\left(\hat{t}_{i}^{m}\right)-p_{i}\left(t_{i}^{0}\right)$, which can be reduced to $u_{\min } \cdot\left(\hat{t}_{i}^{m}\right)^{2}+3 v_{i}\left(t_{i}^{0}\right) \cdot \hat{t}_{i}^{m}-3 L=0$. The solution of the last equation yields $\hat{t}_{i}^{m}=\frac{-3 v_{i}\left(t_{i}^{0}\right) \pm \sqrt{\left.9\left(v_{i}\left(t_{i}^{0}\right)\right)^{2}+12 u_{\min } \cdot L\right)}}{2 u_{\min }}$, from which we have the only admissible result $\hat{t}_{i}^{m}=$ $\frac{-3 v_{i}\left(t_{i}^{0}\right)+\sqrt{\left.9\left(v_{i}\left(t_{i}^{0}\right)\right)^{2}+12 u_{\min } \cdot L\right)}}{2 u_{\min }}$. Hence, for any $t_{i}^{m}$ such that $t_{i}^{m} \geq \hat{t}_{i}^{m}, u_{\min }-u_{i}(t) \leq 0$ becomes active, and the proof of the second statement of Theorem 3 is complete.

\subsection{Interdependence of Constraint Activation Cases}

We have discussed so far the conditions under which any of the state and control constraints become active. Using these conditions, we can derive the analytical solution of (5). However, the resulting solution might activate additional constrained arcs. Therefore, we need to be able to identify beforehand under which conditions any additional constrained arcs may become active. Next, we provide a set of conditions based on the junction point where transition between the constrained and unconstrained arcs occur.

Theorem 4 For $C A V i \in \mathcal{N}(t)$, let $\tau_{s}^{*} \in\left(t_{i}^{0}, t_{i}^{m}\right]$ be the junction point of the state constrained arc where either $v_{i}(t)-v_{\max } \leq 0$ or $v_{\min }-v_{i}(t) \leq 0$ becomes active. Then, (i) $v_{i}(t)-v_{\max } \leq 0$ may cause $u_{i}(t)-u_{\max } \leq 0$ to become active, if $\tau_{s}^{*} \leq$ $\frac{-3 v_{i}\left(t_{i}^{0}\right)+\sqrt{9\left(v_{i}\left(t_{i}^{0}\right)\right)^{2}+12 u_{\max } \cdot\left(p_{i}^{*}\left(\tau_{s}^{*}\right)-p_{i}\left(t_{i}^{0}\right)\right)}}{2 u_{\max }}, \quad$ and

$v_{\min }-v_{i}(t) \leq 0$ may cause $u_{\text {min }}-u_{i}(t) \leq 0$ to become active, if $\tau_{s}^{*} \geq \frac{-3 v_{i}\left(t_{i}^{0}\right)+\sqrt{9\left(v_{i}\left(t_{i}^{0}\right)\right)^{2}+12 u_{\min } \cdot\left(p_{i}^{*}\left(\tau_{s}^{*}\right)-p_{i}\left(t_{i}^{0}\right)\right)}}{2 u_{\min }}$.

PROOF. Suppose that $v_{i}(t)-v_{\max } \leq 0$ becomes active at $\tau_{s}^{*}$, where $t_{i}^{0}<\tau_{s}^{*} \leq t_{i}^{m}$. Then from $(1), u_{i}^{*}(t)=0$ in $t \in\left[\tau_{s}^{*}, t_{i}^{m}\right]$ and $p_{i}\left(\tau_{s}^{*}\right)=p_{i}\left(t_{i}^{m}\right)-v_{\max } \cdot\left(t_{i}^{m}-\tau_{s}^{*}\right)$. We will determine whether any control constraint $u_{i}(t)-u_{\max } \leq$ 0 becomes active in $t \in\left[t_{i}^{0}, \tau_{s}^{*}\right]$. From Lemma 4 , the control constraint becomes active at $t=t_{i}^{0}$. Let $\hat{t}_{i}^{m}$ be the value that $u_{i}(t)-u_{\max } \leq 0$ becomes active at $t_{i}^{0}$, and $\hat{a}_{i}, \hat{b}_{i}$ the corresponding constants of integration. Without loss of generality, if we let $t_{i}^{0}=0$, then from (17) and (19) we can write, $\hat{b}_{i}=-\frac{3\left(v_{i}\left(t_{i}^{0}\right) \cdot \hat{t}_{i}^{m}-\left(p_{i}^{*}\left(\tau_{s}^{*}\right)-p_{i}\left(t_{i}^{0}\right)\right)\right.}{\left(\hat{t}_{i}^{m}\right)^{2}}=$ $u_{\text {max }}$, where $p_{i}\left(\tau_{s}^{*}\right)-p_{i}\left(t_{i}^{0}\right)=p_{i}\left(\hat{t}_{i}^{m}\right)-p_{i}\left(t_{i}^{0}\right)$, which can be reduced to $u_{\max } \cdot\left(\hat{t}_{i}^{m}\right)^{2}+3 v_{i}\left(t_{i}^{0}\right) \cdot \hat{t}_{i}^{m}-3\left(p_{i}^{*}\left(\tau_{s}^{*}\right)-\right.$ $\left.p_{i}\left(t_{i}^{0}\right)\right)=0$. The solution of the last equation yields $\hat{t}_{i}^{m}=$ $\frac{-3 v_{i}\left(t_{i}^{0}\right) \pm \sqrt{\left.9\left(v_{i}\left(t_{i}^{0}\right)\right)^{2}+12 u_{\max } \cdot\left(p_{i}^{*}\left(\tau_{s}^{*}\right)-p_{i}\left(t_{i}^{0}\right)\right)\right)}}{2 u_{\max }}$. Since $\hat{t}_{i}^{m}>0$, $\hat{t}_{i}^{m}=\frac{-3 v_{i}\left(t_{i}^{0}\right)+\sqrt{\left.9\left(v_{i}\left(t_{i}^{0}\right)\right)^{2}+12 u_{\max } \cdot\left(p_{i}^{*}\left(\tau_{s}^{*}\right)-p_{i}\left(t_{i}^{0}\right)\right)\right)}}{2 u_{\max }}$. Hence, for any $\tau_{s}^{*}$ such that $\tau_{s}^{*} \leq \hat{t}_{i}^{m}, u_{i}(t)-u_{\max } \leq 0$ becomes active, and the proof of the first statement of Theorem 4 is complete.

Suppose that $v_{\min }-v_{i}(t) \leq 0$ becomes active at $\tau_{s}^{*}$, where $t_{i}^{0}<\tau_{s}^{*} \leq t_{i}^{m}$. Then from $(1), u_{i}^{*}(t)=0$ in $t \in\left[\tau_{s}^{*}, t_{i}^{m}\right]$ and $p_{i}\left(\tau_{s}^{*}\right)=p_{i}\left(t_{i}^{m}\right)-v_{\min } \cdot\left(t_{i}^{m}-\tau_{s}^{*}\right)$. Let $\hat{t}_{i}^{m}$ be the value that $u_{\text {min }}-u_{i}(t) \leq 0$ becomes active at $t_{i}^{0}$, and $\hat{a}_{i}, \hat{b}_{i}$ the corresponding constants of integration. Without loss of generality, if we let $t_{i}^{0}=0$, then from (17) and (19) we can write, $\hat{b}_{i}=-\frac{3\left(v_{i}\left(t_{i}^{0}\right) \cdot \hat{t}_{i}^{m}-\left(p_{i}^{*}\left(\tau_{s}^{*}\right)-p_{i}\left(t_{i}^{0}\right)\right)\right.}{\left(\hat{t}_{i}^{m}\right)^{2}}=u_{m i n}$, where $p_{i}\left(\tau_{s}^{*}\right)-p_{i}\left(t_{i}^{0}\right)=p_{i}\left(\hat{t}_{i}^{m}\right)-p_{i}\left(t_{i}^{0}\right)$, which can be reduced to $u_{\min } \cdot\left(\hat{t}_{i}^{m}\right)^{2}+3 v_{i}\left(t_{i}^{0}\right) \cdot \hat{t}_{i}^{m}-3\left(p_{i}^{*}\left(\tau_{s}^{*}\right)-\right.$ $\left.p_{i}\left(t_{i}^{0}\right)\right)=0$. The solution of the last equation yields $\hat{t}_{i}^{m}=\frac{-3 v_{i}\left(t_{i}^{0}\right) \pm \sqrt{\left.9\left(v_{i}\left(t_{i}^{0}\right)\right)^{2}+12 u_{\min } \cdot\left(p_{i}^{*}\left(\tau_{s}^{*}\right)-p_{i}\left(t_{i}^{0}\right)\right)\right)}}{2 u_{\min }}$, where $\hat{t}_{i}^{m}=\frac{-3 v_{i}\left(t_{i}^{0}\right)+\sqrt{\left.9\left(v_{i}\left(t_{i}^{0}\right)\right)^{2}+12 u_{\min } \cdot\left(p_{i}^{*}\left(\tau_{s}^{*}\right)-p_{i}\left(t_{i}^{0}\right)\right)\right)}}{2 u_{\min }}$ is the only admissible result. Hence, for any $\tau_{s}^{*}$ such that $\tau_{s}^{*} \geq \hat{t}_{i}^{m}, u_{\min }-u_{i}(t) \leq 0$ becomes active, and the proof of the second statement of Theorem 4 is complete.

Theorem 5 For $C A V i \in \mathcal{N}(t)$, let $\tau_{c}^{*} \in\left(t_{i}^{0}, t_{i}^{m}\right]$ be the junction point of the control constrained arc where either $u_{i}(t)-u_{\max } \leq 0$ or $u_{\min }-u_{i}(t) \leq 0$ becomes active. Then, (i) $u_{i}(t)-u_{\max } \leq 0$ may cause $v_{i}(t)-v_{\max } \leq 0$ to become active, if $t_{i}^{m} \geq \tau_{c}^{*}-\frac{2\left(v_{i}\left(\tau_{c}^{*}\right)-v_{\max }\right)}{u_{\max }}$, and (ii) $u_{\text {min }}-u_{i}(t) \leq 0$ may cause $v_{\min }-v_{i}(t) \leq 0$ to become active, if $t_{i}^{m} \geq \tau_{c}^{*}-\frac{2\left(v_{i}\left(\tau_{c}^{*}\right)-v_{\min }\right)}{u_{m i n}}$.

PROOF. Suppose that $u_{i}(t)-u_{\max } \leq 0$ becomes active at $t_{i}^{0}\left(\right.$ Remark 6) with an exit time at $\tau_{c}^{*} \in\left(t_{i}^{0}, t_{i}^{m}\right]$. Then from (1), $u_{i}^{*}(t)=u_{\max }$ in $t \in\left[t_{i}^{0}, \tau_{c}^{*}\right]$. Consequently, we have $v_{i}\left(\tau_{c}^{*}\right)=v_{i}\left(t_{i}^{0}\right)+u_{\max } \cdot \tau_{c}^{*}$. We will determine whether any state constraint $v_{i}(t)-v_{\max } \leq 0$ becomes active for the unconstrained arc within $t \in$ $\left[\tau_{c}^{*}, t_{i}^{m}\right]$. Suppose that there exists a time $t_{i}^{s} \in\left(\tau_{c}^{*}, t_{i}^{m}\right]$ at which $v_{i}(t)-v_{\max } \leq 0$ becomes active in $\left[\tau_{c}^{*}, t_{i}^{m}\right]$. 
Without loss of generality, if we let $\tau_{c}^{*}=0$, then the constants of integration $\hat{a}_{i}, \hat{b}_{i}$ are given by $\hat{a}_{i}=-\frac{u_{\max }}{\hat{t}_{i}^{m}}$ and $\hat{b}_{i}=u_{\max }\left(\right.$ Remark 2), where $\hat{t}_{i}^{m}:=t_{i}^{m}-\tau_{c}^{*}$. From (15) and (18), we have $\frac{1}{2} \hat{a}_{i} \cdot\left(t_{i}^{s}\right)^{2}+\hat{b}_{i} \cdot t_{i}^{s}+v_{i}\left(\tau_{c}^{*}\right)=$ $v_{\max }$. Solving the quadratic equation for $t_{i}^{s}$, we have $t_{i}^{s}=\frac{-2 \hat{b}_{i} \pm \sqrt{4 \hat{b}_{i}^{2}-8 \hat{a}_{i} \cdot\left(v_{i}\left(\tau_{c}^{*}\right)-v_{\max }\right)}}{2 \hat{a}_{i}}$, which yields $t_{i}^{s}=\hat{t}_{i}^{m} \pm$ $\sqrt{\frac{4 \hat{b}_{i}^{2}-8 \hat{a}_{i} \cdot\left(v_{i}\left(\tau_{c}^{*}\right)-v_{\max }\right)}{4 \hat{a}_{i}^{2}}}$. Since we require $t_{i}^{S} \leq \hat{t}_{i}^{m}$, we need to have $\sqrt{4 \hat{b}_{i}^{2}-8 \hat{a}_{i} \cdot\left(v_{i}\left(\tau_{c}^{*}\right)-v_{\max }\right)} \geq 0$ resulting in $\hat{a}_{i} \leq \frac{2\left(v_{i}\left(\tau_{c}^{*}\right)-v_{\max }\right)}{\left(\hat{t}_{i}^{m}\right)^{2}}$. By using the value of $\hat{a}_{i}$ in the above equation and simplifying, the proof of the first statement of Theorem 2 follows.

For the second statement of Theorem 5, suppose that there exists a time $t_{i}^{s} \in\left(\tau_{c}^{*}, t_{i}^{m}\right]$ at which $v_{\text {min }}-v_{i}(t) \leq 0$ becomes active in $\left[\tau_{c}^{*}, t_{i}^{m}\right]$. Without loss of generality, if we let $\tau_{c}^{*}=0$, then the constants of integration $\hat{a}_{i}, \hat{b}_{i}$ are given by $\hat{a}_{i}=-\frac{u_{\min }}{\hat{t}_{i}^{m}}$ and $\hat{b}_{i}=u_{\min }$ (Remark 2), where $\hat{t}_{i}^{m}:=t_{i}^{m}-\tau_{c}^{*}$. From (15) and (18), we have $\frac{1}{2} \hat{a}_{i} \cdot\left(t_{i}^{s}\right)^{2}+$ $\hat{b}_{i} \cdot t_{i}^{s}+v_{i}\left(\tau_{c}^{*}\right)=v_{\text {min }}$. Solving the quadratic equation for $t_{i}^{s}$, we have $t_{i}^{s}=\frac{-2 \hat{b}_{i} \pm \sqrt{4 \hat{b}_{i}^{2}-8 \hat{a}_{i} \cdot\left(v_{i}\left(\tau_{c}^{*}\right)-v_{\text {min }}\right)}}{2 \hat{a}_{i}}$, which yields $t_{i}^{s}=\hat{t}_{i}^{m} \pm \sqrt{\frac{4 \hat{b}_{i}^{2}-8 \hat{a}_{i} \cdot\left(v_{i}\left(\tau_{c}^{*}\right)-v_{\text {min }}\right)}{4 \hat{a}_{i}^{2}}}$. Since $t_{i}^{s} \leq \hat{t}_{i}^{m}$, we need to have $\sqrt{4 \hat{b}_{i}^{2}-8 \hat{a}_{i} \cdot\left(v_{i}\left(\tau_{c}^{*}\right)-v_{\text {min }}\right)} \geq 0$ resulting in $\hat{a}_{i} \leq \frac{2\left(v_{i}\left(\tau_{c}^{*}\right)-v_{\min }\right)}{\left(\hat{t}_{i}^{m}\right)^{2}}$. By using the value of $\hat{a}_{i}$ in the above equation and simplifying, the proof of the second statement of Theorem 2 follows.

Remark 7 The conditions in Theorems 4 and 5 depend on the junction points $\tau_{s}^{*}$ and $\tau_{c}^{*}$ of the corresponding constraint activation cases, which can be derived analytically from the known boundary conditions of (5). Since the derivation of such analytical solution requires additional information, we provide the analysis in the following section.

\section{Analytical Solution of the Constrained Opti- mal Control Problem}

To derive the analytical solution of (5), we present a condition-based framework consisting of the following steps. We first evaluate the condition stated in Theorem 1 to reduce the set of possible constraint activation cases (Remark 5). Then using above result, we evaluate the conditions presented in Theorems 2 and 3 to determine whether any constraint has become active. If none of the constraints in (2) becomes active, we simply derive the unconstrained solution using (14)-(16) and terminate the process. However, if the conditions in Theorems 2 and 3 indicate the activation of any constraint cases, we need to evaluate further the conditions in Theorems 4 and 5 to determine whether any additional constraints may become active within the constrained solution as a result of the constraint cases identified from Theorems 2 and 3 . Once the nature of the final constraint activation case is identified using Theorems 4 and 5, we then piece together the relevant unconstrained and constrained arcs that yield a set of algebraic equations which are solved simultaneously using the boundary conditions of (5) and interior conditions between the arcs.

Since we piece together multiple constrained and unconstrained arcs, we denote the constants of integration corresponding to each arc by $a_{i}^{(p)}, b_{i}^{(p)}, c_{i}^{(p)}, d_{i}^{(p)}, p=$ $1,2, \ldots, N_{\text {arc }}$, where $N_{\text {arc }} \in \mathbb{N}$ is the total number of arcs pieced together in the constrained solution and $p$ represents the position of the arcs in terms of their appearance in the optimal solution starting from $t_{i}^{0}$ to $t_{i}^{m}$. For $N_{\text {arc }}$ arcs, we have $\left(N_{\text {arc }}-1\right)$ junction points. At any junction point $\tau$, the states are continuous, namely,

$$
p_{i}\left(\tau^{-}\right)=p_{i}\left(\tau^{+}\right), v_{i}\left(\tau^{-}\right)=v_{i}\left(\tau^{+}\right),
$$

where, $\tau^{-}$and $\tau^{+}$represent the time instance right before and right after $\tau$, respectively.

In what follows, we present the closed form analytical solution of different cases of state and control constraint activation to derive the optimal input $u_{i}^{*}(t), t \in\left[t_{i}^{0}, t_{i}^{m}\right]$, for each CAV $i \in \mathcal{N}(t)$.

Case 1 Only the state constraint $v_{i}(t)-v_{\max } \leq 0$ becomes active.

In this case, we have $\mu_{i}^{a}(t)=\mu_{i}^{b}(t)=\eta_{i}^{d}(t)=0$. From (11), (12), and (13), we have $u_{i}(t)+\lambda_{i}^{v}(t)=0, \dot{\lambda}_{i}^{p}(t)=$ 0 , and $\dot{\lambda}_{i}^{v}(t)=-\lambda_{i}^{p}(t)-\eta_{i}^{c}(t)$. By Lemma 5, CAV $i \in$ $\mathcal{N}(t)$ exits the constrained arc at $t=t_{i}^{m}$ which leads to a single junction point. Let $\tau_{s}, t_{i}^{0}<\tau_{s}<t_{i}^{m}$, be the junction point and let $\tau_{s}^{-}$and $\tau_{s}^{+}$be the time instance just before and after time $\tau_{s}$. The optimal speed and control input on the constrained arc are

$$
v_{i}^{*}(t)=v_{\max }, u_{i}^{*}(t)=0, t \in\left[\tau_{s}, t_{i}^{m}\right] .
$$

The jump conditions of the costates and the Hamiltonian at $\tau_{s}$ are

$$
\begin{aligned}
& \lambda_{i}^{p}\left(\tau_{s}^{-}\right)=\lambda_{i}^{p}\left(\tau_{s}^{+}\right)+\left.\pi_{i} \cdot \frac{\partial}{\partial p_{i}(t)}\left[v_{i}(t)-v_{\max }\right]\right|_{t=\tau_{s}}, \\
& \lambda_{i}^{v}\left(\tau_{s}^{-}\right)=\lambda_{i}^{v}\left(\tau_{s}^{+}\right)+\left.\pi_{i} \cdot \frac{\partial}{\partial v_{i}(t)}\left[v_{i}(t)-v_{\max }\right]\right|_{t=\tau_{s}}, \\
& H_{i}\left(\tau_{s}^{-}\right)=H_{i}\left(\tau_{s}^{+}\right)-\left.\pi_{i} \cdot \frac{\partial}{\partial t}\left[v_{i}(t)-v_{\max }\right]\right|_{t=\tau_{s}},
\end{aligned}
$$


where $\pi_{i}$ is a constant Langrange multiplier determined so that $v_{i}(t)-v_{\max }=0$ is satisfied. Note that, (23a)(23c) imply possible discontinuity of the costates and the Hamiltonian at $t=\tau_{s}$. The state variables are continuous at $t=\tau_{s}$. From (23c), we have

$$
\begin{gathered}
\frac{1}{2} u_{i}^{2}\left(\tau_{s}^{-}\right)+\lambda_{i}^{p}\left(\tau_{s}^{-}\right) \cdot v_{i}\left(\tau_{s}^{-}\right)+\lambda_{i}^{v}\left(\tau_{s}^{-}\right) \cdot u_{i}\left(\tau_{s}^{-}\right) \\
+\eta_{i}^{c}\left(\tau_{s}^{-}\right) \cdot\left(v_{i}\left(\tau_{s}^{-}\right)-v_{\max }\right)=\frac{1}{2} u_{i}^{2}\left(\tau_{s}^{+}\right)+\lambda_{i}^{p}\left(\tau_{s}^{+}\right) \cdot v_{i}\left(\tau_{s}^{+}\right) \\
+\lambda_{i}^{v}\left(\tau_{s}^{+}\right) \cdot u_{i}\left(\tau_{s}^{+}\right)+\eta_{i}^{c}\left(\tau_{s}^{+}\right) \cdot\left(v_{i}\left(\tau_{s}^{+}\right)-v_{\max }\right) .
\end{gathered}
$$

From the continuity of the states and since $v_{i}\left(\tau_{s}^{+}\right)=$ $v_{\max }, u_{i}\left(\tau_{s}^{+}\right)=0$, we have $\lambda_{i}^{p}\left(\tau_{s}^{-}\right) \cdot v_{i}\left(\tau_{s}^{-}\right)=\lambda_{i}^{p}\left(\tau_{s}^{+}\right)$. $v_{i}\left(\tau_{s}^{+}\right)$. The Lagrange multiplier $\eta_{i}^{c}(t)$ in $(9)$, yields $\eta_{i}^{c}\left(\tau_{s}^{-}\right) \cdot\left(v_{i}\left(\tau_{s}^{-}\right)-v_{\max }\right)=\eta_{i}^{c}\left(\tau_{s}^{+}\right) \cdot\left(v_{i}\left(\tau_{s}^{+}\right)-v_{\max }\right)=$ 0 . By combining the above equations, (24) reduces to $\frac{1}{2} u_{i}^{2}\left(\tau_{s}^{-}\right)+\lambda_{i}^{v}\left(\tau_{s}^{-}\right) \cdot u_{i}\left(\tau_{s}^{-}\right)=0$, which implies that either $u_{i}\left(\tau_{s}^{-}\right)=0$ or $\frac{1}{2} u_{i}\left(\tau_{s}^{-}\right)+\lambda_{i}^{v}\left(\tau_{s}^{-}\right)=0$, or both. Since the second term contradicts $u_{i}(t)+\lambda_{i}^{v}(t)=0$, we have $u_{i}\left(\tau_{s}^{-}\right)=0$. The Lagrange multiplier $\eta_{i}^{c}(t)$ is $\eta_{i}^{c}(t)= \begin{cases}0, & \text { if } v_{i}(t)<v_{\max }, t \in\left[t_{i}^{0}, \tau_{s}\right), \\ -\lambda_{i}^{p}(t), & \text { if } v_{i}(t)=v_{\max }, t \in\left[\tau_{s}, t_{i}^{m}\right] .\end{cases}$

Using the Euler-Lagrange equations, interior conditions, the initial and final boundary conditions, and the terminal condition of the costates, we can formulate a set of equations by piecing the unconstrained and constrained arcs together at time $t=\tau_{s}$. This results in a total number of 9 equations that we need to solve simultaneously to compute $4+4+1=9$ variables corresponding to the constants of integration of unconstrained and constrained arc, and the junction point $\tau_{s}^{*}$ respectively. From (14)-(16) and the boundary conditions in (5), we receive the following 4 equations: $\frac{1}{2} a_{i}^{(1)} \cdot\left(t_{i}^{0}\right)^{2}+b_{i}^{(1)} \cdot t_{i}^{0}+c_{i}^{(1)}=v_{i}\left(t_{i}^{0}\right), \frac{1}{6} a_{i}^{(1)} \cdot\left(t_{i}^{0}\right)^{3}+$ $\frac{1}{2} b_{i}^{(1)} \cdot\left(t_{i}^{0}\right)^{2}+c_{i}^{(1)} \cdot t_{i}^{0}+d_{i}^{(1)}=p_{i}\left(t_{i}^{0}\right), a_{i}^{(2)} \cdot t_{i}^{m}+b_{i}^{(2)}=$ $0, \frac{1}{6} a_{i}^{(2)} \cdot\left(t_{i}^{m}\right)^{3}+\frac{1}{2} b_{i}^{(2)} \cdot\left(t_{i}^{m}\right)^{2}+c_{i}^{(2)} \cdot t_{i}^{m}+d_{i}^{(2)}=p_{i}\left(t_{i}^{m}\right)$. From the state and control continuity at the junction point $\tau_{s}$, we receive the remaining 5 equations are,

$$
\begin{aligned}
& \frac{1}{2} a_{i}^{(1)} \cdot\left(\tau_{s}\right)^{2}+b_{i}^{(1)} \cdot \tau_{s}+c_{i}^{(1)}=v_{\max }, \\
& a_{i}^{(1)} \cdot \tau_{s}+b_{i}^{(1)}=0 \\
& \frac{1}{6} a_{i}^{(1)} \cdot\left(\tau_{s}\right)^{3}+\frac{1}{2} b_{i}^{(1)} \cdot\left(\tau_{s}\right)^{2}+c_{i}^{(1)} \cdot \tau_{s}+d_{i}^{(1)} \\
& +v_{\max } \cdot\left(t_{i}^{m}-\tau_{s}\right)=p_{i}\left(t_{i}^{m}\right), \\
& \frac{1}{2} a_{i}^{(2)} \cdot\left(\tau_{s}\right)^{2}+b_{i}^{(2)} \cdot \tau_{s}+c_{i}^{(2)}=v_{\max }, \\
& a_{i}^{(2)} \cdot \tau_{s}+b_{i}^{(2)}=0,
\end{aligned}
$$

where $a_{i}^{(1)}, b_{i}^{(1)}, c_{i}^{(1)}, d_{i}^{(1)}$ and $a_{i}^{(2)}, b_{i}^{(2)}, c_{i}^{(2)}, d_{i}^{(2)}$ are the constants of integration for the unconstrained and constrained arcs, respectively. The recursive process to solve the above set of equations cannot be computed in real time. Additionally, the computational speed and convergence of numerical methods are also sensitive to the initial guess of the variables, which impose additional burden on the real-time computation effort. However, if the junction point $\tau_{s}^{*}$ can be derived as an explicit function of the initial and final boundary conditions, then the above set of equations can lead to a closed-form solution that can be solved analytically in real time.

Lemma 6 For $C A V i \in \mathcal{N}(t)$, let $\tau_{s}^{*}$ be the junction point between the unconstrained and constrained arc of the state constrained $v_{i}(t)-v_{\max } \leq 0$ solution. Then $\tau_{s}^{*}$ is an explicit function of $p_{i}\left(t_{i}^{m}\right), \bar{v}_{\max }, t_{i}^{m}$, and $v_{i}\left(t_{i}^{0}\right)$, and can be expressed as $\tau_{s}^{*}=\frac{3\left(p_{i}\left(t_{i}^{m}\right)-v_{\max } \cdot t_{i}^{m}\right)}{\left(v_{i}\left(t_{i}^{0}\right)-v_{\max }\right)}$.

PROOF. See Appendix A.

Case 2 Only the control constraint $u_{i}(t)-u_{\max } \leq 0$ becomes active.

In this case, we have $\mu_{i}^{b}(t)=\eta_{i}^{c}(t)=\eta_{i}^{d}(t)=0$. From (11), (12), and (13), we have $u_{i}(t)+\lambda_{i}^{v}(t)+\mu_{i}^{a}(t)=$ $0, \dot{\lambda}_{i}^{p}(t)=0$, and $\dot{\lambda}_{i}^{v}(t)=-\lambda_{i}^{p}(t)$. By Lemma 4, CAV $i \in \mathcal{N}(t)$ enters the constrained arc at time $t=t_{i}^{0}$ and has a single exit junction point. Let $\tau_{c}, t_{i}^{0}<\tau_{c}<t_{i}^{m}$, be the junction point where the control constrained arc transitions into the unconstrained arc, and let $\tau_{c}^{-}$and $\tau_{c}^{+}$be the immediate left and the right instance of $\tau_{c}$. The optimal control input $u_{i}^{*}(t)$ at the junction point is $u_{i}^{*}\left(\tau_{c}\right)=u_{\max }$. The jump conditions are $\lambda_{i}^{p}\left(\tau_{c}^{-}\right)-\lambda_{i}^{p}\left(\tau_{c}^{+}\right)=0, \lambda_{i}^{v}\left(\tau_{c}^{-}\right)-\lambda_{i}^{v}\left(\tau_{c}^{+}\right)=$ 0 , and $H_{i}\left(\tau_{c}^{+}\right)-H_{i}\left(\tau_{c}^{-}\right)=0$, which imply continuity of the costates and the Hamiltonian at the junction point $t=\tau_{c}$. The last jump condition leads to $\frac{1}{2} u_{i}^{2}\left(\tau_{c}^{-}\right)+\lambda_{i}^{p}\left(\tau_{c}^{-}\right) \cdot v_{i}\left(\tau_{c}^{-}\right)+\lambda_{i}^{v}\left(\tau_{c}^{-}\right) \cdot u_{i}\left(\tau_{c}^{-}\right)+$ $\mu_{i}^{a}\left(\tau_{c}^{-}\right) \cdot\left(u_{i}\left(\tau_{c}^{-}\right)-u_{\max }\right)=\frac{1}{2} u_{i}^{2}\left(\tau_{c}^{+}\right)+\lambda_{i}^{p}\left(\tau_{s}^{+}\right) \cdot$ $v_{i}\left(\tau_{c}^{+}\right)+\lambda_{i}^{v}\left(\tau_{c}^{+}\right) \cdot u_{i}\left(\tau_{c}^{+}\right)+\mu_{i}^{a}\left(\tau_{c}^{+}\right) \cdot\left(u_{i}\left(\tau_{c}^{+}\right)-u_{\max }\right)$. From the continuity of the state and costate $\lambda_{i}^{p}$ at $t=\tau_{c}$, we have $\lambda_{i}^{p}\left(\tau_{c}^{-}\right) \cdot v_{i}\left(\tau_{c}^{-}\right)=\lambda_{i}^{p}\left(\tau_{c}^{+}\right) \cdot v_{i}\left(\tau_{c}^{+}\right)$. Moreover, (7) yields $\mu_{i}^{a}\left(\tau_{c}^{-}\right) \cdot\left(u_{i}\left(\tau_{c}^{-}\right)-u_{\max }\right)=$ $\mu_{i}^{a}\left(\tau_{c}^{+}\right) \cdot\left(u_{i}\left(\tau_{c}^{+}\right)-u_{\max }\right)=0$, which after simplification leads to either $u_{i}\left(\tau_{c}^{+}\right)=u_{i}\left(\tau_{c}^{-}\right)$or $\frac{1}{2}\left(u_{i}\left(\tau_{c}^{+}\right)+\right.$ $\left.u_{i}\left(\tau_{c}^{-}\right)\right)+\lambda_{i}^{v}\left(\tau_{c}^{+}\right)=0$, or both. Both equations lead to $u_{i}\left(\tau_{c}^{+}\right)=u_{i}\left(\tau_{c}^{-}\right)=u_{\max }$. The Lagrange multiplier $\mu_{i}^{a}(t)$ is $\mu_{i}^{a}(t)= \begin{cases}-\lambda_{i}^{v}(t)-u_{\max }, & \text { if } t \in\left[t_{i}^{0}, \tau_{c}\right), \\ 0, & \text { if } t \in\left[\tau_{c}, t_{i}^{m}\right] .\end{cases}$

Using the Euler-Lagrange equations, jump conditions at the junction point, the initial and final boundary conditions, and the costate condition at $t=t_{i}^{m}$, we can formulate a set of equations by piecing the constrained and unconstrained arcs together at $t=\tau_{c}$. In this case, we have a constrained arc with constant parameters $a_{i}^{(1)}, b_{i}^{(1)}, c_{i}^{(1)}, d_{i}^{(1)}$, followed by an unconstrained arc 
with constant parameters $a_{i}^{(2)}, b_{i}^{(2)}, c_{i}^{(2)}, d_{i}^{(2)}$ pieced together at junction point $\tau_{c}$, leading to $4+4+1=9$ variables that need to be determined. At time $t=t_{i}^{0}$ and $t=\tau_{c}$, we have the following set of equations for the constrained arc,

$$
\begin{aligned}
& a_{i}^{(1)} \cdot t_{i}^{0}+b_{i}^{(1)}=u_{\max } \\
& a_{i}^{(1)} \cdot \tau_{c}+b_{i}^{(1)}=u_{\max } \\
& \frac{1}{2} a_{i}^{(1)} \cdot\left(t_{i}^{0}\right)^{2}+b_{i}^{(1)} \cdot t_{i}^{0}+c_{i}^{(1)}=v_{i}\left(t_{i}^{0}\right), \\
& \frac{1}{6} a_{i}^{(1)} \cdot\left(t_{i}^{0}\right)^{3}+\frac{1}{2} b_{i}^{(1)} \cdot\left(t_{i}^{0}\right)^{2}+c_{i}^{(1)} \cdot t_{i}^{0}+d_{i}^{(1)}=p_{i}\left(t_{i}^{0}\right) .
\end{aligned}
$$

From (26a) and (26b), considering $t_{i}^{0}=0$ without loss of generality, we have $a_{i}^{(1)}=0$ and $b_{i}^{(1)}=u_{\max }$. Substituting in (26c), we have $c_{i}^{(1)}=v_{i}\left(t_{i}^{0}\right)$. Finally, solving $(26 \mathrm{~d}), d_{i}^{(1)}=p_{i}\left(t_{i}^{0}\right)$. The following set of equations aim to determine the remaining constants of integration $a_{i}^{(2)}, b_{i}^{(2)}, c_{i}^{(2)}, d_{i}^{(2)}$ of the exiting unconstrained arc and the junction point $\tau_{c}^{*}$

$$
\begin{aligned}
& a_{i}^{(2)} \cdot \tau_{c}+b_{i}^{(2)}=u_{\max }, \\
& a_{i}^{(2)} \cdot t_{i}^{m}+b_{i}^{(2)}=0, \\
& \frac{1}{2} a_{i}^{(2)} \cdot \tau_{c}^{2}+\left(b_{i}^{(2)}-u_{\max }\right) \cdot \tau_{c}+c_{i}^{(2)}-v_{i}^{0}=0, \\
& \frac{1}{6} a_{i}^{(2)} \cdot \tau_{c}^{3}+\frac{1}{2}\left(b_{i}^{(2)}-u_{\max }\right) \cdot \tau_{c}^{2}+\left(c_{i}^{(2)}-v_{i}^{0}\right) \cdot \tau_{c} \\
& +d_{i}^{(2)}-p_{i}\left(t_{i}^{0}\right)=0, \\
& \frac{1}{6} a_{i}^{(2)} \cdot\left(t_{i}^{m}\right)^{3}+\frac{1}{2} b_{i}^{(2)} \cdot\left(t_{i}^{m}\right)^{2}+c_{i}^{(2)} \cdot t_{i}^{m}+d_{i}^{(2)}=p_{i}\left(t_{i}^{m}\right) .
\end{aligned}
$$

Lemma 7 For $C A V i \in \mathcal{N}(t)$, let $\tau_{c}^{*}$ be the junction point between the unconstrained and control constraint $u_{i}(t)-u_{\max } \leq 0$ solution. Then $\tau_{c}^{*}$ can be expressed as an explicit function of $p_{i}\left(t_{i}^{m}\right), p_{i}\left(t_{i}^{0}\right), u_{\max }, t_{i}^{m}$, and $v_{i}\left(t_{i}^{0}\right)$.

PROOF. See Appendix B.

Case 3 Both state constraint $v_{i}(t)-v_{\max } \leq 0$ and the control constraint $u_{i}(t)-u_{\max } \leq 0$ become active.

If both $u_{i}(t)-u_{\max } \leq 0$ and $v_{i}(t)-v_{\max } \leq 0$ become active, we derive the analytical solution combining the steps described in the previous two cases. In this case, we have $\mu_{i}^{b}(t)=\eta_{i}^{d}(t)=0$. From (11), (12), and (13), we have $u_{i}(t)+\lambda_{i}^{v}(t)+\mu_{i}^{a}(t)=0, \dot{\lambda}_{i}^{p}(t)=$ 0 , and $\dot{\lambda}_{i}^{v}(t)=-\lambda_{i}^{p}(t)-\eta_{i}^{c}(t)$. Let $\tau_{c}$ be the junction point that $\mathrm{CAV} i \in \mathcal{N}(t)$ exits the control constrained arc and $\tau_{s}$ be the junction point that CAV $i$ enters the state constrained arc such that $t_{i}^{0}<\tau_{c}<\tau_{s}<t_{i}^{m}$. The optimal control input at the control constrained arc is $u_{i}^{*}(t)=u_{\max }$, for all $t \in\left[t_{i}^{0}, \tau_{c}\right]$. In the state constrained arc, we have $v_{i}^{*}(t)=v_{\max }, u_{i}^{*}(t)=0$, for all $t \in\left[\tau_{s}, t_{i}^{m}\right]$. From the jump conditions at the junction points $\tau_{c}$ and $\tau_{s}$, we have continuity in the state and control input. The Lagrange multipliers $\mu_{i}^{a}(t)$ and $\eta_{i}^{c}(t)$ are given by

$$
\begin{aligned}
& \mu_{i}^{a}(t)=\left\{\begin{array}{ll}
0, & t \in\left(\tau_{c}, t_{i}^{m}\right], \\
-\lambda_{i}^{v}(t)-u_{\max }, & t \in\left[t_{i}^{0}, \tau_{c}\right],
\end{array}\right\}, \text { and } \\
& \eta_{i}^{c}(t)=\left\{\begin{array}{ll}
0, & t \in\left[t_{i}^{0}, \tau_{s}\right), \\
-\lambda_{i}^{p}(t), & t \in\left[\tau_{s}, t_{i}^{m}\right] .
\end{array}\right\} .
\end{aligned}
$$

Solving (26a)-(26d), considering $t_{i}^{0}=0$ without loss of generality, the constants of integration $a_{i}^{(1)}, b_{i}^{(1)}, c_{i}^{(1)}, d_{i}^{(1)}$ of the control constrained arc are $a_{i}^{(1)}=0, b_{i}^{(1)}=$ $u_{\max }, c_{i}^{(1)}=v_{i}^{0}$ and $d_{i}^{(1)}=p_{i}\left(t_{i}^{0}\right)$. The unconstrained arc with constants of integration $a_{i}^{(2)}, b_{i}^{(2)}, c_{i}^{(2)}$, and $d_{i}^{(2)}$ can consists of the following set of equations,

$$
\begin{aligned}
& a_{i}^{(2)} \cdot \tau_{c}+b_{i}^{(2)}=u_{\max } \\
& \frac{1}{2} a_{i}^{(2)} \cdot \tau_{c}^{2}+\left(b_{i}^{(2)}-u_{\max }\right) \cdot \tau_{c}+c_{i}^{(2)}-v_{i}^{0}=0 \\
& \frac{1}{6} a_{i}^{(2)} \cdot \tau_{c}^{3}+\frac{1}{2}\left(b_{i}^{(2)}-u_{\max }\right) \cdot \tau_{c}^{2}+\left(c_{i}^{(2)}-v_{i}^{0}\right) \cdot \tau_{c} \\
& +\left(d_{i}^{(2)}-p_{i}\left(t_{i}^{0}\right)\right)=0 \\
& a_{i}^{(2)} \cdot \tau_{s}+b_{i}^{(2)}=0 \\
& \frac{1}{2} a_{i}^{(2)} \cdot \tau_{s}^{2}+b_{i}^{(2)} \cdot \tau_{s}+c_{i}^{(2)}-v_{\max }=0 \\
& \frac{1}{6} a_{i}^{(2)} \cdot\left(\tau_{s}\right)^{3}+\frac{1}{2} b_{i}^{(2)} \cdot\left(\tau_{s}\right)^{2}+c_{i}^{(2)} \cdot \tau_{s}+d_{i}^{(2)} \\
& +v_{\max } \cdot\left(t_{i}^{m}-\tau_{s}\right)=p_{i}\left(t_{i}^{m}\right)
\end{aligned}
$$

Finally, the state-constrained arc with constants of integration $a_{i}^{(3)}, b_{i}^{(3)}, c_{i}^{(3)}, d_{i}^{(3)}$ consists of the following set of equations,

$$
\begin{aligned}
& a_{i}^{(3)} \cdot t_{i}^{m}+b_{i}^{(3)}=0, \\
& a_{i}^{(3)} \cdot \tau_{s}+b_{i}^{(3)}=0 \\
& \frac{1}{2} a_{i}^{(3)} \cdot \tau_{s}^{2}-b_{i}^{(3)} \cdot \tau_{s}-c_{i}^{(3)}-v_{\max }=0 \\
& \frac{1}{6} a_{i}^{(3)} \cdot\left(t_{i}^{m}\right)^{3}+\frac{1}{2} b_{i}^{(3)} \cdot\left(t_{i}^{m}\right)^{2}+c_{i}^{(3)} \cdot t_{i}^{m} \\
& +d_{i}^{(3)}-p_{i}\left(t_{i}^{m}\right)=0 .
\end{aligned}
$$

From (29a)-(29d), we have $a_{i}^{(3)}=0, b_{i}^{(3)}=0, c_{i}^{(3)}=$ $v_{\max }$ and $d_{i}^{(3)}=p_{i}\left(t_{i}^{m}\right)-v_{\max } \cdot t_{i}^{m}$. The remaining constants of integration $a_{i}^{(2)}, b_{i}^{(2)}, c_{i}^{(2)}, d_{i}^{(2)}$ of the unconstrained arc, and the junction points $\tau_{s}^{*}$ and $\tau_{c}^{*}$ can be determined by solving the set of equations (28a)-(28f).

Lemma 8 The junction point $\tau_{s}^{*}$ between the unconstrained and the constrained arc if $v_{i}(t)-v_{\max } \leq$ 
0 becomes active, and the junction point $\tau_{c}^{*}$ between the unconstrained and the constrained arc if $u_{i}(t)-u_{\max } \leq 0$ also becomes active are explicit functions of $p_{i}\left(t_{i}^{m}\right), v_{\max }, u_{\max }, t_{i}^{m}$, and $v_{i}\left(t_{i}^{0}\right)$.

PROOF. See Appendix C.

Case 4 Only the state constraint $v_{\text {min }}-v_{i}(t) \leq 0$ becomes active.

In this case, we have $\mu_{i}^{a}(t)=\mu_{i}^{b}(t)=\eta_{i}^{c}(t)=0$. From (11), (12), and (13), we have $u_{i}(t)+\lambda_{i}^{v}(t)=0, \dot{\lambda}_{i}^{p}(t)=$ 0 , and $\dot{\lambda}_{i}^{v}(t)=-\lambda_{i}^{p}(t)-\eta_{i}^{d}(t)$. Let $t=\tau_{s}$ be the junction point that $v_{\min }-v_{i}(t) \leq 0$ becomes active. The optimal speed and control at the junction point are $v_{i}^{*}(t)=$ $v_{m i n}, u_{i}^{*}(t)=0$, for all $t \in\left[\tau_{s}, t_{i}^{m}\right]$. The jump conditions are

$$
\begin{aligned}
& \lambda_{i}^{p}\left(\tau_{s}^{-}\right)=\lambda_{i}^{p}\left(\tau_{s}^{+}\right)+\left.\pi_{i} \cdot \frac{\partial}{\partial p_{i}(t)}\left[v_{\text {min }}-v_{i}(t)\right]\right|_{t=\tau_{s}}, \\
& \lambda_{i}^{v}\left(\tau_{s}^{-}\right)=\lambda_{i}^{v}\left(\tau_{s}^{+}\right)+\left.\pi_{i} \cdot \frac{\partial}{\partial v_{i}(t)}\left[v_{\text {min }}-v_{i}(t)\right]\right|_{t=\tau_{s}}, \\
& H_{i}\left(\tau_{s}^{-}\right)=H_{i}\left(\tau_{s}^{+}\right)-\left.\pi_{i} \cdot \frac{\partial}{\partial t}\left[v_{\text {min }}-v_{i}(t)\right]\right|_{t=\tau_{s}},
\end{aligned}
$$

where $\pi_{i}$ is a constant Langrange multiplier determined so that $v_{\text {min }}-v_{i}(t)=0$ is satisfied. Note that, (30a)(30c) imply possible discontinuity of the costates and the Hamiltonian at $t=\tau_{s}$. The state variables are continuous at $t=\tau_{s}$. From (30a) and (30c), the position costate and the Lagrangian of the Hamiltonian is continuous at $t=\tau_{s}$.

Lemma 9 If the state constraint $v_{\min }-v_{i}(t) \leq 0$ becomes active, then the control input $u_{i}(t)$ is continuous at the junction point $t=\tau_{s}$.

PROOF. See Appendix D.

The Lagrange multiplier $\eta_{i}^{d}(t)$ can be expressed as, $\eta_{i}^{d}(t)=\left\{\begin{array}{ll}0, & \text { if } \quad t \in\left[t_{i}^{0}, \tau_{s}\right), \\ -\lambda_{i}^{p}(t), & \text { if } \quad t \in\left[\tau_{s}, t_{i}^{m}\right] .\end{array}\right.$ Using the EulerLagrange equations, interior conditions, initial and final boundary conditions, and the costate condition at $t=t_{i}^{m}$, we can formulate a set of equations similar to Case 1 to solve for $4+4+1=9$ variables corresponding to the constants of integration of the unconstrained and constrained arc, and the junction point $\tau_{s}$. The set of equations of the unconstrained arc with constants of integration $a_{i}^{(1)}, b_{i}^{(1)}, c_{i}^{(1)}, d_{i}^{(1)}$ are, $\frac{1}{2} a_{i}^{(1)} \cdot\left(t_{i}^{0}\right)^{2}+b_{i}^{(1)} \cdot t_{i}^{0}+c_{i}^{(1)}=v_{i}\left(t_{i}^{0}\right), \frac{1}{6} a_{i}^{(1)} \cdot\left(t_{i}^{0}\right)^{3}+\frac{1}{2} b_{i}^{(1)}$. $\left(t_{i}^{0}\right)^{2}+c_{i}^{(1)} \cdot t_{i}^{0}+d_{i}^{(1)}=p_{i}\left(t_{i}^{0}\right), \frac{1}{2} a_{i}^{(1)} \cdot\left(\tau_{s}\right)^{2}+b_{i}^{(1)} \cdot \tau_{s}+c_{i}^{(1)}=$ $v_{\text {min }}, a_{i}^{(1)} \cdot \tau_{s}+b_{i}^{(1)}=0$, and $\frac{1}{6} a_{i}^{(1)} \cdot\left(\tau_{s}\right)^{3}+\frac{1}{2} b_{i}^{(1)} \cdot\left(\tau_{s}\right)^{2}+$ $c_{i}^{(1)} \cdot \tau_{s}+d_{i}^{(1)}+v_{\text {min }} \cdot\left(t_{i}^{m}-\tau_{s}\right)=p_{i}\left(t_{i}^{m}\right)$. The set of equations of the state constrained arc with the constants of integration $a_{i}^{(2)}, b_{i}^{(2)}, c_{i}^{(2)}, d_{i}^{(2)}$ are $\frac{1}{2} a_{i}^{(2)} \cdot\left(\tau_{s}\right)^{2}+b_{i}^{(2)}$. $\tau_{s}+c_{i}^{(2)}=v_{\min }, a_{i}^{(2)} \cdot t_{i}^{m}+b_{i}^{(2)}=0, a_{i}^{(2)} \cdot \tau_{s}+b_{i}^{(2)}=$ 0 , and $\frac{1}{6} a_{i}^{(2)} \cdot\left(t_{i}^{0}\right)^{3}+\frac{1}{2} b_{i}^{(2)} \cdot\left(t_{i}^{0}\right)^{2}+c_{i}^{(2)} \cdot t_{i}^{0}+d_{i}^{(2)}=p_{i}\left(t_{i}^{m}\right)$, which yield $a_{i}^{(2)}=0, b_{i}^{(2)}=0, c_{i}^{(2)}=v_{\min }$ and $d_{i}^{(2)}=p_{i}\left(t_{i}^{m}\right)-v_{\text {min }} \cdot t_{i}^{m}$. The remaining constants of integration $a_{i}^{(1)}, b_{i}^{(1)}, c_{i}^{(1)}, d_{i}^{(1)}$ and the junction point $\tau_{s}^{*}$ can be determined numerically by solving simultaneously the above set of equations.

Lemma 10 For $C A V i \in \mathcal{N}(t)$, let $\tau_{s}^{*}$ be the junction point between the unconstrained and constrained arc of the state constrained $v_{\text {min }}-v_{i}(t) \leq 0$ solution. Then $\tau_{s}^{*}$ is an explicit function of $p_{i}\left(t_{i}^{m}\right), v_{\text {min }}, t_{i}^{m}$ and $v_{i}\left(t_{i}^{0}\right)^{s}$, and can be expressed as $\tau_{s}^{*}=\frac{3\left(p_{i}\left(t_{i}^{m}\right)-v_{\min } \cdot t_{i}^{m}\right)}{\left(v_{i}\left(t_{i}^{0}\right)-v_{m i n}\right)}$

PROOF. The proof is similar to the proof of Lemma 6 (see Appendix A), hence it is omitted.

Case 5 Only the control constraint $u_{m i n}-u_{i}(t) \leq 0$ becomes active.

In this case, we have $\mu_{i}^{a}(t)=\eta_{i}^{c}(t)=\eta_{i}^{d}(t)=0$. From (11), (12), and (13), we have $u_{i}(t)+\lambda_{i}^{v}(t)-\mu_{i}^{b}(t)=$ $0, \dot{\lambda}_{i}^{p}(t)=0$, and $\dot{\lambda}_{i}^{v}(t)=-\lambda_{i}^{p}(t)$. Let $\tau_{c}>t_{i}^{0}$ be the junction point that CAV $i \in \mathcal{N}(t)$ transitions from the constrained arc to the unconstrained arc. The optimal control at the junction point $\tau_{c}$ is $u_{i}^{*}\left(\tau_{c}\right)=u_{m i n}$. From the jump conditions, we have $\lambda_{i}^{p}\left(\tau_{c}^{-}\right)=\lambda_{i}^{p}\left(\tau_{c}^{+}\right)$, $\lambda_{i}^{v}\left(\tau_{c}^{-}\right)=\lambda_{i}^{v}\left(\tau_{c}^{+}\right)$, and $H_{i}\left(\tau_{c}^{+}\right)=H_{i}\left(\tau_{c}^{-}\right)$.

Lemma 11 If the control constraint $u_{\min }-u_{i}(t) \leq 0$ becomes active, then the control input $u(t)$ is continuous at the junction point $t=\tau_{c}$.

PROOF. See Appendix E.

The Lagrange multiplier $\mu_{i}^{b}(t)$ can be expressed as, $\mu_{i}^{b}(t)=\left\{\begin{array}{ll}\lambda_{i}^{v}(t)+u_{\text {min }}, & \text { if } t \in\left[t_{i}^{0}, \tau_{c}\right), \\ 0, & \text { if } t \in\left[\tau_{c}, t_{i}^{m}\right] .\end{array}\right.$ Using the Euler-Lagrange equations, interior condition, initial and final boundary conditions, and the condition of costates at $t=t_{i}^{m}$, we have a set of equations of the constrained arc: $a_{i}^{(1)} \cdot t_{i}^{0}+b_{i}^{(1)}=u_{m i n}, a_{i}^{(1)} \cdot \tau_{c}+b_{i}^{(1)}=$ $u_{\text {min }}, \frac{1}{2} a_{i}^{(1)}\left(t_{i}^{0}\right)^{2}+b_{i}^{(1)} \cdot t_{i}^{0}+c_{i}^{(1)}=v_{i}\left(t_{i}^{0}\right)$, and $\frac{1}{6} a_{i}^{(1)}\left(t_{i}^{0}\right)^{3}+$ $\frac{1}{2} b_{i}^{(1)} \cdot\left(t_{i}^{0}\right)^{2}+c_{i}^{(1)} \cdot t_{i}^{0}+d_{i}^{(1)}=0$, resolving which 
with $t_{i}^{0}=0$ yields, $a_{i}^{(1)}=0, b_{i}^{(1)}=u_{\min }, c_{i}^{(1)}=$ $v_{i}\left(t_{i}^{0}\right), d_{i}^{(1)}=p_{i}\left(t_{i}^{0}\right)$, where $a_{i}^{(1)}, b_{i}^{(1)}, c_{i}^{(1)}, d_{i}^{(1)}$ are the constants of integration for the constrained arc. In addition, we have a set of equations of the unconstrained arc: $a_{i}^{(2)} \cdot \tau_{c}-b_{i}^{(2)}+u_{\min }=0, a_{i}^{(2)} \cdot t_{i}^{m}+b_{i}^{(2)}=$ $0, \frac{1}{2} a_{i}^{(2)} \cdot \tau_{c}^{2}+\left(b_{i}^{(2)}-u_{m i n}\right) \cdot \tau_{c}+c_{i}^{(2)}-v_{i}\left(t_{i}^{0}\right)=0$, $\frac{1}{6} a_{i}^{(2)} \cdot \tau_{c}^{3}+\frac{1}{2}\left(b_{i}^{(2)}-u_{\min }\right) \cdot \tau_{c}+\left(c_{i}^{(2)}-v_{i}\left(t_{i}^{0}\right) \cdot \tau_{c}+d_{i}^{(2)}=\right.$ 0 , and $\frac{1}{6} a_{i}^{(2)} \cdot\left(t_{i}^{m}\right)^{3}+\frac{1}{2} b_{i}^{(2)} \cdot\left(t_{i}^{m}\right)^{2}+c_{i}^{(2)} \cdot t_{i}^{m}+d_{i}^{(2)}-$ $p_{i}\left(t_{i}^{m}\right)=0$, where $a_{i}^{(2)}, b_{i}^{(2)}, c_{i}^{(2)}, d_{i}^{(2)}$ are the constants of integration of the unconstrained arc.

Lemma 12 For $C A V i \in \mathcal{N}(t)$, let $\tau_{s}^{*}$ be the junction point between the unconstrained and constrained arc of the control constrained $\left(u_{\text {min }}-u_{i}(t) \leq 0\right)$ solution of (5). Then $\tau_{c}^{*}$ can be expressed as an explicit function of $p_{i}\left(t_{i}^{m}\right), p_{i}\left(t_{i}^{0}\right), u_{m i n}, t_{i}^{m}$, and $v_{i}\left(t_{i}^{0}\right)$.

PROOF. The proof is similar to the proof of Lemma 7 (see Appendix B), hence it is omitted.

Case 6 Both state constraint $v_{\min }-v_{i}(t) \leq 0$ and the control constraint $u_{\text {min }}-u_{i}(t) \leq 0$ become active.

In this case, we can derive the analytical solution following similar steps to Case 3. A control constrained $u_{\min }-u_{i}(t) \leq 0$ arc with constants of integration $a_{i}^{(1)}, b_{i}^{(1)}, c_{i}^{(1)}, d_{i}^{(1)}$ is pieced together with an unconstrained arc with constants of integration $a_{i}^{(2)}, b_{i}^{(2)}, c_{i}^{(2)}, d_{i}^{(2)}$ at the junction point $\tau_{c}$. The unconstrained arc is pieced together with the state constrained $v_{\text {min }}-v_{i}(t) \leq 0$ arc with constants of integration $a_{i}^{(3)}, b_{i}^{(3)}, c_{i}^{(3)}, d_{i}^{(3)}$ at the junction point $\tau_{s}$. The constants of integration of the constrained and unconstrained arcs, and the junction points $\tau_{s}^{*}$ and $\tau_{c}^{*}$ can be determined by a set of equations similar to those derived in Case 3.

Lemma 13 The junction point $\tau_{s}^{*}$ between the unconstrained and the constrained arc when $v_{\min }-v_{i}(t) \leq 0$ becomes active, and the junction point $\tau_{c}^{*}$ between the unconstrained and the constrained arc when $u_{\text {min }}-u_{i}(t) \leq 0$ also becomes active are explicit functions of $p_{i}\left(t_{i}^{m}\right), v_{\text {min }}, u_{m i n}, t_{i}^{m}$, and $v_{i}\left(t_{i}^{0}\right)$.

PROOF. The proof is similar to the proof of Lemma 8 (see Appendix E), hence it is omitted.

\section{Simulation Results}

We validate the analytical solution of the optimal control problem (5) through numerical simulation in MATLAB. In this section, we present the results considering $t_{i}^{m}=$ $10 \mathrm{~s}$, where only the state constraint $v_{i}(t)-v_{\max } \leq 0$ and control constraint $u_{i}(t)-u_{\max } \leq 0$ can become active
(Theorem 1). Similar results to those presented here can be also derived for the case where $v_{\text {min }}-v_{i}(t) \leq 0$ and $u_{\text {min }}-u_{i}(t) \leq 0$ become active. We consider the initial and final position of CAV $i \in \mathcal{N}(t)$ to be $p_{i}\left(t_{i}^{0}\right)=0 \mathrm{~m}$ and $p_{i}\left(t_{i}^{m}\right)=200 \mathrm{~m}$, and the initial speed $v_{i}\left(t_{i}^{0}\right)=14.3 \mathrm{~m} / \mathrm{s}$. For each CAV $i \in \mathcal{N}(t)$, we enforce the maximum speed limit and acceleration to be $v_{\max }=22 \mathrm{~m} / \mathrm{s}$ and $u_{\max }=$ $1.8 \mathrm{~m} / \mathrm{s}^{2}$ respectively. The standard procedure to solve

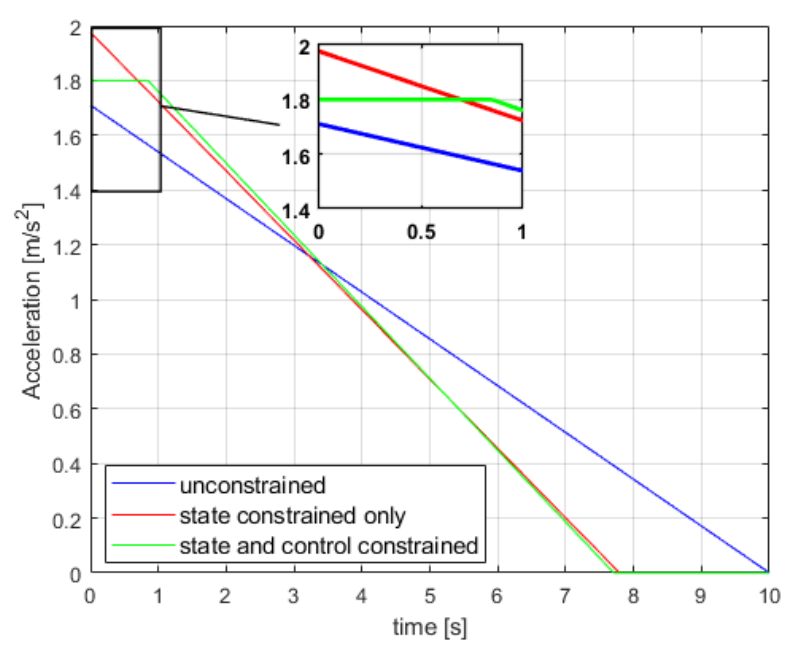

Fig. 2. Optimal control trajectory for the unconstrained (blue), state constraint $v_{i}(t)-v_{\max } \leq 0$ only (red) and both state-control constraint (green) case.

the optimal control problem (5) is to identify whether any of the state or control constraints become active and derive the constrained solution in a recursive manner until none of the constraints are active, as shown in Fig. 2. The unconstrained solution (blue trajectory in Fig. 2 ) activates the state constraint $v_{i}(t)-v_{\max } \leq 0$ only. The acceleration corresponding to the state-constrained $\left(v_{i}(t)-v_{\max } \leq 0\right)$ solution is shown by the red trajectory in Fig. 2, where the unconstrained and constrained arcs are pieced together at the junction point at $t=7.79 \mathrm{~s}$. However, the state-constrained solution (red trajectory in Fig. 2) has to be re-derived since the control constraint $u_{i}(t)-u_{\max } \leq 0$, which was not active before, becomes active now as shown by the red trajectory in Fig. 2 . The constrained optimal control input is derived by piecing the state and control constrained arcs together, and it is shown by the green trajectory in Fig. 2 .

In our condition-based framework, we do not need to consider the intermediate iterative steps above, i.e., the unconstrained (blue trajectory) and state constrained solution (red trajectory) in Fig. 2. We can directly derive the final closed-form analytical solution (green trajectory in Fig. 2) by sequentially checking the conditions in Theorems 1-5. First, we start with Theorem 1 to reduce the possible constraint activation set. Since the first statement of Theorem 1 holds for $t_{i}^{m}=10 \mathrm{~s}$ and the boundary conditions, we only need to consider whether 
$v_{i}(t)-v_{\max } \leq 0$ or $u_{i}(t)-u_{\max } \leq 0$ become active, which reduces the possible constraint activation cases from 15 to 3 . Then, we use Theorems 2 and 3 to identify the specific constraint activation case. In this case, part (i) of Theorem 2 holds, indicating that $v_{i}(t)-v_{\max } \leq 0$ becomes active in $\left(t_{i}^{0}, t_{i}^{m}\right]$. However, part (i) of Theorem 3 does not hold indicating that $u_{i}(t)-u_{\max } \leq 0$ will not become active. Using the result obtained above, we then check part (i) of Theorem 4 which readily indicates that an additional and initially non-existent control constraint $u_{i}(t)-u_{\max } \leq 0$ becomes active within the state-constrained solution, as shown by the red trajectory in Fig. 2. Using the result of Theorem 4, we apply the analysis presented in Case 3 to determine the complete state and control constrained-optimal solution. Here, the aforementioned condition-based framework requires $0.001107 \mathrm{~s}$ to solve in an Intel Core i7-6700 CPU @ $3.40 \mathrm{GHz}$ using MATLAB R2017b. Note that, if the first statement of Theorem 4 does not hold, then none of the control constraints can become active, and thus we can use the analysis presented in Case 1 to determine the optimal solution.

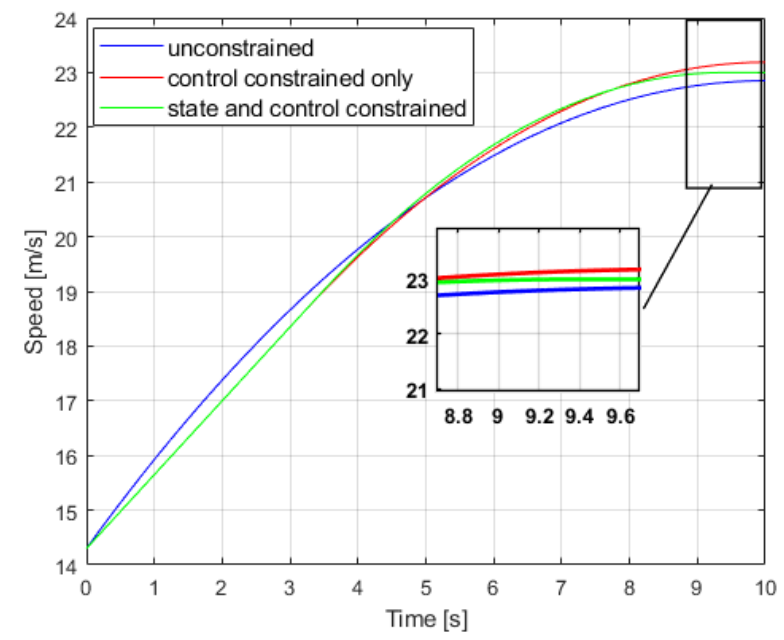

Fig. 3. Optimal speed trajectory for the unconstrained (blue), control constraint $u_{i}(t)-u_{\max } \leq 0$ only (red) and both state-control constraint (green) case.

Next, we consider a different scenario to show the impact when the control constraint $\left(u_{i}(t)-u_{\max } \leq 0\right)$ becomes active (Fig. 3). In this case, we set the maximum speed $v_{\max }$ and acceleration $u_{\max }$ to be $23 \mathrm{~m} / \mathrm{s}$ and $1.35 \mathrm{~m} / \mathrm{s}^{2}$ respectively. Following the above procedure, we check part (i) of Theorem 2 and 3. Since only part (i) of Theorem 3 holds, we conclude that the control constraint $u_{i}(t)-u_{\max } \leq 0$ will become active. We then check part (i) of Theorem 5 to check whether any additional state constraint will become active within the control constrained solution. In this case, part (i) of Theorem 5 holds, as evident from the control constrained state trajectory (red trajectory) in Fig. 3. Therefore, we use the analysis presented in Case 3 to derive the complete state- and control- constrained solution as illustrated by the green trajectory in Fig. 3. Note that, in Fig. 3, in the unconstrained solution (blue trajectory) none of the state constraints become active. However, the controlconstrained solution (red trajectory) activates the state constraint $v_{i}(t)-v_{\max } \leq 0$. Based on our conditionbased framework, we can avoid the computation of the intermediate solutions, i.e., the unconstrained trajectory (blue trajectory in Fig. 3) and the control constrained trajectory (red trajectory in Fig. 3), and directly derive the final constrained trajectory as illustrated by the green trajectory in Fig. 3.

\section{Concluding Remarks}

In this paper, we addressed the state and control constrained optimal framework for coordinating CAVs at different traffic scenarios such as merging at roadways and roundabouts, cruising in congested traffic, passing through speed reduction zones, under 100\% CAV penetration, and provided a condition-based framework to determine the constrained solution without requiring to follow the standard recursive process. We mathematically characterized the activation cases of different state and control constraint combinations, and provided a priori conditions under which different constraint combination can become active. In addition, we presented the closedform analytical solution of the constrained optimal control problem that can be derived and implemented in real time. We validated a subset of constraint activation cases through numerical simulation and showed how the proposed framework can identify the interdependent constraint activation based on the boundary conditions. By eliminating the intermediate steps of solving the constrained optimal control problem, the proposed condition-based framework improves on the standard methodology to solve the constrained optimal control problem.

The proposed framework has certain limitations since it does not consider the optimal control problem with constrained terminal speed, which may result in multiple junction points leading to a more complex formulation. Moreover, in our framework, we considered $100 \%$ penetration rate of CAVs having access to perfect information (no errors or delays) which both impose limitations for real-world applications. It is expected that CAVs will gradually penetrate the market, interact with nonCAVs and contend with vehicle-to-vehicle and vehicleto infrastructure communication limitations, e.g., bandwidth, dropouts, errors and/or delays. Ongoing work includes further exposition into the the existence of the optimal solution under different constraint combinations, and the consideration of the terminal speed constrained formulation. Future work should also address the implementation of the proposed framework under different penetration rates of CAVs and imperfect communication. 


\section{Appendix}

\section{A Proof of Lemma 6}

If $v_{i}(t)-v_{\max } \leq 0$ becomes active, we have an unconstrained arc (with constant parameters $a_{i}^{(1)}, b_{i}^{(1)}, c_{i}^{(1)}, d_{i}^{(1)}$ ) followed by a constrained arc (with constant parameters $a_{i}^{(2)}, b_{i}^{(2)}, c_{i}^{(2)}, d_{i}^{(2)}$ ) pieced together at the junction point $t=\tau_{s}^{*}$. The constrained arc yields at $t=\tau_{s}^{*}$ and $t=t_{i}^{m}$,

$$
\begin{aligned}
& a_{i}^{(2)} \cdot \tau_{s}^{*}+b_{i}^{(2)}=0 \\
& a_{i}^{(2)} \cdot t_{i}^{m}+b_{i}^{(2)}=0 \\
& \frac{1}{2} a_{i}^{(2)} \cdot\left(t_{i}^{m}\right)^{2}+b_{i}^{(2)} \cdot\left(t_{i}^{m}\right)+c_{i}^{(2)}=v_{\max }, \\
& \frac{1}{6} a_{i}^{(2)} \cdot\left(\tau_{s}^{*}\right)^{3}+\frac{1}{2} b_{i}^{(2)} \cdot\left(\tau_{s}^{*}\right)^{2}+c_{i}^{(2)} \cdot\left(\tau_{s}^{*}\right)+d_{i}^{(2)} \\
& \quad+v_{\max } \cdot\left(t_{i}^{m}-\tau_{s}^{*}\right)=p_{i}\left(t_{i}^{m}\right) .
\end{aligned}
$$

From (A.1a) and (A.1b), we have $a_{i}^{(2)}=0$ and $b_{i}^{(2)}=0$. Substituting in (A.1c), we have $c_{i}^{(2)}=v_{\max }$. Finally, from (A.1d) we have $d_{i}^{(2)}=\left(p_{i}\left(t_{i}^{m}\right)-v_{\max } \cdot t_{i}^{m}\right)$. The unconstrained arc at the initial condition $t=t_{i}^{0}$ yields the following equations: $\frac{1}{2} a_{i}^{(1)} \cdot\left(t_{i}^{0}\right)^{2}+b_{i}^{(1)} \cdot\left(t_{i}^{0}\right)+c_{i}^{(2)}=$ $v_{i}\left(t_{i}^{0}\right), \frac{1}{6} a_{i}^{(1)} \cdot\left(t_{i}^{0}\right)^{3}+\frac{1}{2} b_{i}^{(1)} \cdot\left(t_{i}^{0}\right)^{2}+c_{i}^{(1)} \cdot\left(t_{i}^{0}\right)+d_{i}^{(1)}=$ $p_{i}\left(t_{i}^{0}\right)$. Solving the above two equations by considering $t_{i}^{0}=0$, without loss of generality, we have $c_{i}^{(1)}=v_{i}\left(t_{i}^{0}\right)$ and $d_{i}^{(1)}=0$. At $\tau_{s}^{*}$, we have the following set of equations for the unconstrained arc,

$$
\begin{aligned}
& a_{i}^{(1)} \cdot \tau_{s}^{*}+b_{i}^{(1)}=0, \\
& \frac{1}{2} a_{i}^{(1)} \cdot\left(\tau_{s}^{*}\right)^{2}+b_{i}^{(1)} \cdot \tau_{s}^{*}+\left(v_{i}\left(t_{i}^{0}\right)-v_{\max }\right)=0, \\
& \frac{1}{6} a_{i}^{(1)} \cdot\left(\tau_{s}^{*}\right)^{3}+\frac{1}{2} b_{i}^{(1)} \cdot\left(\tau_{s}^{*}\right)^{2} \\
& +\left(v_{i}\left(t_{i}^{0}\right)-v_{\max }\right) \cdot \tau_{s}^{*}-\left(p_{i}\left(t_{i}^{m}\right)-v_{\max } \cdot t_{i}^{m}\right)=0 .
\end{aligned}
$$

Substituting $\tau_{s}^{*}=-\frac{b_{i}^{(1)}}{a_{i}^{(1)}}$ from (A.2a) in (A.2b), we have $\frac{\left(b_{i}^{(1)}\right)^{2}}{a_{i}^{(1)}}=2\left(v_{i}\left(t_{i}^{0}\right)-v_{\max }\right)$. Substituting $\tau_{s}^{*}=-\frac{b_{i}^{(1)}}{a_{i}^{(1)}}$ from (A.2a) in (A.2c), we have $\frac{1}{3} \frac{\left(b_{i}^{(1)}\right)^{3}}{\left(a_{i}^{(1)}\right)^{2}}+\frac{\left(b_{i}^{(1)}\right)}{a_{i}^{(1)}} \cdot\left(v_{\max }-\right.$ $\left.v_{i}\left(t_{i}^{0}\right)\right)-\left(p_{i}\left(t_{i}^{m}\right)-v_{\max } \cdot t_{i}^{m}\right)=0$. From the last two equations, we obtain $\tau_{s}^{*}=-\frac{3\left(p_{i}\left(t_{i}^{m}\right)-v_{\max } \cdot t_{i}^{m}\right)}{\left(v_{\max }-v_{i}\left(t_{i}^{0}\right)\right)}$, where $\tau_{s}^{*}$ is an explicit function of the known parameters $p_{i}\left(t_{i}^{m}\right), v_{\max }, v_{i}\left(t_{i}^{0}\right)$ and $t_{i}^{m}$.

\section{B Proof of Lemma 7}

If $u_{i}(t)-u_{\max } \leq 0$ becomes active, we have a constrained $\operatorname{arc}$ (with constant parameters $a_{i}^{(1)}, b_{i}^{(1)}, c_{i}^{(1)}, d_{i}^{(1)}$ ) followed by an unconstrained arc (with constant parameters $\left.a_{i}^{(2)}, b_{i}^{(2)}, c_{i}^{(2)}, d_{i}^{(2)}\right)$ pieced together at the junction point $t=\tau_{c}^{*}$. Solving (27a) and (27c)$(27 \mathrm{e})$, we have $a_{i}^{(2)}=-\sqrt{\frac{\left(u_{\max }\right)^{3}}{3\left(t_{i}^{m}\right)^{2} \cdot u_{\max }+6 t_{i}^{m} \cdot v_{i}\left(t_{i}^{0}\right)-6 L}}$, where $L=p_{i}\left(t_{i}^{m}\right)-p_{i}\left(t_{i}^{0}\right)$. From (27a) and (27b), $\tau_{c}^{*}=\frac{u_{\max }}{a_{i}^{(2)}}+t_{i}^{m}$. Finally, substituting $a_{i}^{(2)}$ into the last equation, the junction point $\tau_{c}^{*}$ is given by $\tau_{c}^{*}=t_{i}^{m}-\frac{u_{\max }}{\sqrt{\frac{\left(u_{\max }\right)^{3}}{3\left(t_{i}^{m}\right)^{2} \cdot u_{\max }+6 t_{i}^{m} \cdot v_{i}\left(t_{i}^{0}\right)-6 L}}}$, and can be simplified to $\tau_{c}^{*}=t_{i}^{m}-\sqrt{\frac{3\left(t_{i}^{m}\right)^{2} \cdot u_{\max }+6 t_{i}^{m} \cdot v_{i}\left(t_{i}^{0}\right)-6 L}{u_{\max }}}$, which is an explicit function of the known boundary parameters $t_{i}^{m}, p_{i}\left(t_{i}^{m}\right), p_{i}\left(t_{i}^{0}\right), v_{i}\left(t_{i}^{0}\right)$, and $u_{\max }$.

\section{Proof of Lemma 8}

If $u_{i}(t)-u_{\max } \leq 0$ becomes active, we have a constrained arc with constants of integration $a_{i}^{(1)}, b_{i}^{(1)}, c_{i}^{(1)}, d_{i}^{(1)}$ followed by an unconstrained arc with constants of integration $a_{i}^{(2)}, b_{i}^{(2)}, c_{i}^{(2)}, d_{i}^{(2)}$, pieced together at the junction point $t=\tau_{c}^{*}$. If $v_{i}(t)-v_{\max } \leq 0$ becomes active, we have a constrained arc with constants of integration $a_{i}^{(2)}, b_{i}^{(2)}, c_{i}^{(2)}, d_{i}^{(2)}$ followed by a constrained arc with constants of integration $a_{i}^{(3)}, b_{i}^{(3)}, c_{i}^{(3)}, d_{i}^{(3)}$ pieced together at the junction point $t=\tau_{s}^{*}$. Solving (26a)-(26d) for the control constrained arc with $t_{i}^{0}=0$, we have $a_{i}^{(1)}=0, b_{i}^{(1)}=u_{\max }, c_{i}^{(1)}=v_{i}\left(t_{i}^{0}\right)$ and $d_{i}^{(1)}=p_{i}\left(t_{i}^{0}\right)$. Solving (29a)-(29d) for the state constrained arc, considering $t_{i}^{0}=0$ without loss of generality, we have $a_{i}^{(3)}=0, b_{i}^{(3)}=0, c_{i}^{(3)}=v_{\max }$ and $d_{i}^{(3)}=p_{i}\left(t_{i}^{m}\right)-v_{\max } \cdot t_{i}^{m}$. From (28a) and (28d), we have $\tau_{c}^{*}=\frac{u_{\max }-b_{i}^{(2)}}{a_{i}^{(2)}}$ and $\tau_{s}^{*}=-\frac{b_{i}^{(2)}}{a_{i}^{(2)}}$ respectively. Substituting the latter into (28b), (28c), (28e) and (28f), and solving the system of equations, we have $a_{i}^{(2)}=-u_{\text {max }}^{2}$.

$\sqrt{-\frac{1}{\phi}}$, and $b_{i}^{(2)}=\frac{u_{\max }\left(-2 v_{i}\left(t_{i}^{0}\right) \sqrt{-\frac{1}{\phi}}+2 v_{\max } \sqrt{-\frac{1}{\phi}}+1\right)}{2}$, where, $\phi\left(t_{i}^{m}, p_{i}\left(t_{i}^{m}\right), v_{i}\left(t_{i}^{0}\right), u_{\max }, v_{\max }\right)=-24\left(t_{i}^{m}\right.$. $\left.u_{\max } \cdot v_{\max }-p_{i}\left(t_{i}^{m}\right) \cdot u_{\max }+v_{i}\left(t_{i}^{0}\right) \cdot v_{\max }\right)+12\left(v_{i}^{2}\left(t_{i}^{0}\right)+\right.$ $\left.v_{\max }^{2}\right)$. Substituting the last results into (28a) and (28d), the junction points $\tau_{s}^{*}$ and $\tau_{c}^{*}$ are given as explicit functions of the known parameters $t_{i}^{m}, p_{i}\left(t_{i}^{m}\right), v_{i}\left(t_{i}^{0}\right), u_{\max }$ and $v_{\max }$.

\section{Proof of Lemma 9}

From (30c), we have

$$
\begin{gathered}
\frac{1}{2} u_{i}^{2}\left(\tau_{s}^{-}\right)+\lambda_{i}^{p}\left(\tau_{s}^{-}\right) \cdot v_{i}\left(\tau_{s}^{-}\right)+\lambda_{i}^{v}\left(\tau_{s}^{-}\right) \cdot u_{i}\left(\tau_{s}^{-}\right) \\
+\eta_{i}^{d}\left(\tau_{s}^{-}\right) \cdot\left(v_{\min }-v_{i}\left(\tau_{s}^{-}\right)\right)=\frac{1}{2} u_{i}^{2}\left(\tau_{s}^{+}\right)+\lambda_{i}^{p}\left(\tau_{s}^{+}\right) \cdot v_{i}\left(\tau_{s}^{+}\right) \\
+\lambda_{i}^{v}\left(\tau_{s}^{+}\right) \cdot u_{i}\left(\tau_{s}^{+}\right)+\eta_{i}^{d}\left(\tau_{s}^{+}\right) \cdot\left(v_{\min }-v_{i}\left(\tau_{s}^{+}\right)\right) . \quad(\mathrm{D} .1)
\end{gathered}
$$


Since $v_{i}\left(\tau_{s}^{+}\right)=v_{\min }$ and $u_{i}\left(\tau_{s}^{+}\right)=0$, and from the continuity of state $(21)$ and $\lambda_{i}^{p}(30 \mathrm{a})$, we have $\lambda_{i}^{p}\left(\tau_{s}^{-}\right) \cdot v_{i}\left(\tau_{s}^{-}\right)=$ $\lambda_{i}^{p}\left(\tau_{s}^{+}\right) \cdot v_{i}\left(\tau_{s}^{+}\right)$. From $(9)$, we have $\eta_{i}^{d}\left(\tau_{s}^{-}\right) \cdot\left(v_{\min }-\right.$ $\left.v_{i}\left(\tau_{s}^{-}\right)\right)=\eta_{i}^{d}\left(\tau_{s}^{+}\right) \cdot\left(v_{\text {min }}-v_{i}\left(\tau_{s}^{+}\right)\right)=0$. Hence, (D.1) reduces to $\frac{1}{2} u_{i}^{2}\left(\tau_{s}^{-}\right)+\lambda_{i}^{v}\left(\tau_{s}^{-}\right) \cdot u_{i}\left(\tau_{s}^{-}\right)=0$, which implies that either $u_{i}\left(\tau_{s}^{-}\right)=0$ or $\frac{1}{2} u_{i}\left(\tau_{s}^{-}\right)+\lambda_{i}^{v}\left(\tau_{s}^{-}\right)=0$, or both. Since the second term can not hold, we have $u_{i}\left(\tau_{s}^{-}\right)=u_{i}\left(\tau_{s}^{+}\right)=0$.

\section{E Proof of Lemma 11}

Since $H_{i}\left(\tau_{c}^{+}\right)=H_{i}\left(\tau_{c}^{-}\right)$, we have $\frac{1}{2} u_{i}^{2}\left(\tau_{c}^{-}\right)+\lambda_{i}^{p}\left(\tau_{c}^{-}\right)$. $v_{i}\left(\tau_{c}^{-}\right)+\lambda_{i}^{v}\left(\tau_{c}^{-}\right) \cdot u_{i}\left(\tau_{c}^{-}\right)+\mu_{i}^{b}\left(\tau_{c}^{-}\right) \cdot\left(u_{\min }-u_{i}\left(\tau_{c}^{-}\right)\right)=$ $\frac{1}{2} u_{i}^{2}\left(\tau_{c}^{+}\right)+\lambda_{i}^{p}\left(\tau_{s}^{+}\right) \cdot v_{i}\left(\tau_{c}^{+}\right)+\lambda_{i}^{v}\left(\tau_{c}^{+}\right) \cdot u_{i}\left(\tau_{c}^{+}\right)+\mu_{i}^{b}\left(\tau_{c}^{+}\right) \cdot$ $\left(u_{\text {min }}-u_{i}\left(\tau_{c}^{+}\right)\right)$. From the continuity of the state $(21)$ and $\lambda_{i}^{p}$ at $t=\tau_{c}$, we have $\lambda_{i}^{p}\left(\tau_{c}^{-}\right) \cdot v_{i}\left(\tau_{c}^{-}\right)=\lambda_{i}^{p}\left(\tau_{c}^{+}\right) \cdot v_{i}\left(\tau_{c}^{+}\right)$. From (7) we have $\mu_{i}^{b}\left(\tau_{c}^{-}\right) \cdot\left(u_{\min }-u_{i}\left(\tau_{c}^{-}\right)\right)=\mu_{i}^{b}\left(\tau_{c}^{+}\right)$. $\left(u_{\text {min }}-u_{i}\left(\tau_{c}^{+}\right)\right)=0$. After simplifying, we have either $u_{i}\left(\tau_{c}^{+}\right)=u_{i}\left(\tau_{c}^{-}\right)$or $\frac{1}{2}\left(u_{i}\left(\tau_{c}^{+}\right)+u_{i}\left(\tau_{c}^{-}\right)\right)+\lambda_{i}^{v}\left(\tau_{c}^{+}\right)=0$. Both the equations lead to the continuity in control input $u_{i}(t)$ at time $t=\tau_{c}$, i.e., $u_{i}\left(\tau_{c}^{+}\right)=u_{i}\left(\tau_{c}^{-}\right)$.

\section{References}

J. Alonso, V. Milanés, J. Pérez, E. Onieva, C. González, and T. de Pedro. Autonomous vehicle control systems for safe crossroads. Transportation Research Part C: Emerging Technologies, 19(6):1095-1110, Dec. 2011.

M. Athans. A unified approach to the vehicle-merging problem. Transportation Research, 3(1):123-133, 1969. ISSN 00411647.

T.-C. Au and P. Stone. Motion Planning Algorithms for Autonomous Intersection Management. In $A A A I$ 2010 Workshop on Bridging the Gap Between Task and Motion Planning (BTAMP), 2010.

A. Bakibillah, M. Kamal, C. Tan, et al. The optimal coordination of connected and automated vehicles at roundabouts. In 2019 58th Annual Conference of the Society of Instrument and Control Engineers of Japan (SICE), pages 1392-1397. IEEE, 2019.

A. E. Bryson and Y. C. Ho. Applied optimal control: optimization, estimation and control. CRC Press, 1975.

A. E. Bryson, Jr., W. F. Denham, and S. E. Dreyfus. Optimal programming problems with inequality constraints I: Necessary conditions for extremal solutions. American Institute of Aeronautics and Astronautics Journal, 1(11):2544 - 2550, 1963.

A. de La Fortelle. Analysis of reservation algorithms for cooperative planning at intersections. 13th International IEEE Conference on Intelligent Transportation Systems, pages 445-449, Sept. 2010.

K. Dresner and P. Stone. Multiagent traffic management: a reservation-based intersection control mechanism. In Proceedings of the Third International Joint Conference on Autonomous Agents and Multiagents Systems, pages 530-537, 2004.
K. Dresner and P. Stone. A multiagent approach to autonomous intersection management. Journal of artificial intelligence research, 31:591-656, 2008.

J. Guanetti, Y. Kim, and F. Borrelli. Control of Connected and Automated Vehicles: State of the Art and Future Challenges. Annual Reviews in Control, 45:1840, 2018.

J. Han, A. Sciarretta, L. L. Ojeda, G. De Nunzio, and L. Thibault. Safe-and eco-driving control for connected and automated electric vehicles using analytical state-constrained optimal solution. IEEE Transactions on Intelligent Vehicles, 3(2):163-172, 2018.

S. Huang, A. Sadek, and Y. Zhao. Assessing the Mobility and Environmental Benefits of Reservation-Based Intelligent Intersections Using an Integrated Simulator. IEEE Transactions on Intelligent Transportation Systems, 13(3):1201-1214, 2012.

Z. Huang, W. Zhuang, G. Yin, L. Xu, and K. Luo. Cooperative merging for multiple connected and automated vehicles at highway on-ramps via virtual platoon formation. In 2019 Chinese Control Conference (CCC), pages 6709-6714. IEEE, 2019.

M. Kamal, J. Imura, a. Ohata, T. Hayakawa, and K. Aihara. Coordination of automated vehicles at a trafficlightless intersection. 16th International IEEE Conference on Intelligent Transportation Systems (ITSC 2013), (Itsc):922-927, Oct. 2013.

K.-D. Kim and P. Kumar. An MPC-Based Approach to Provable System-Wide Safety and Liveness of Autonomous Ground Traffic. IEEE Transactions on Automatic Control, 59(12):3341-3356, 2014.

W. Levine and M. Athans. On the optimal error regulation of a string of moving vehicles. IEEE Transactions on Automatic Control, 11(3):355-361, 1966.

X.-Y. Lu and J. K. Hedrick. Longitudinal control algorithm for automated vehicle merging. International Journal of Control, 76(2):193-202, 2003.

A. I. Mahbub, A. A. Malikopoulos, and L. Zhao. Decentralized optimal coordination of connected and automated vehicles for multiple traffic scenarios. Automatica, 117:108958, 2020a.

A. M. I. Mahbub and A. A. Malikopoulos. Conditions for state and control constraint activation in coordination of connected and automated vehicles. Proceedings of 2020 American Control Conference, pages 436-441, 2020 .

A. M. I. Mahbub, L. Zhao, D. Assanis, and A. A. Malikopoulos. Energy-Optimal Coordination of Connected and Automated Vehicles at Multiple Intersections. In Proceedings of 2019 American Control Conference, pages 2664-2669, 2019.

A. M. I. Mahbub, V. Karri, D. Parikh, S. Jade, and A. Malikopoulos. A decentralized time- and energyoptimal control framework for connected automated vehicles: From simulation to field test. In $S A E$ Technical Paper 2020-01-0579. SAE International, 2020b.

A. M. I. Mahbub, A. Malikopoulos, and L. Zhao. Impact of connected and automated vehicles in a corridor. In Proceedings of 2020 American Control Conference, 
2020, pages 1185-1190. IEEE, 2020c.

G. Mahler and A. Vahidi. An optimal velocity-planning scheme for vehicle energy efficiency through probabilistic prediction of traffic-signal timing. IEEE Transactions on Intelligent Transportation Systems, 15(6): 2516-2523, 2014.

L. Makarem and D. Gillet. Fluent coordination of autonomous vehicles at intersections. 2012 IEEE International Conference on Systems, Man, and Cybernetics (SMC), pages 2557-2562, Oct. 2012.

A. A. Malikopoulos and J. P. Aguilar. An Optimization Framework for Driver Feedback Systems. IEEE Transactions on Intelligent Transportation Systems, 14(2): 955-964, 2013.

A. A. Malikopoulos and L. Zhao. Optimal path planning for connected and automated vehicles at urban intersections. In Proceedings of the 58th IEEE Conference on Decision and Control, 2019, pages 1261-1266. IEEE, 2019a.

A. A. Malikopoulos and L. Zhao. A closed-form analytical solution for optimal coordination of connected and automated vehicles. In 2019 American Control Conference $(A C C)$, pages 3599-3604. IEEE, 2019b.

A. A. Malikopoulos, C. G. Cassandras, and Y. J. Zhang. A decentralized energy-optimal control framework for connected automated vehicles at signal-free intersections. Automatica, 93:244-256, 2018.

A. A. Malikopoulos, S. Hong, B. Park, J. Lee, and S. Ryu. Optimal control for speed harmonization of automated vehicles. IEEE Transactions on Intelligent Transportation Systems, 20(7):2405-2417, 2019.

A. A. Malikopoulos, L. E. Beaver, and I. V. Chremos. Optimal time trajectory and coordination for connected and automated vehicles. Automatica, 125: 109469, 2021.

R. Margiotta and D. Snyder. An agency guide on how to establish localized congestion mitigation programs. Technical report, U.S. Department of Transportation. Federal Highway Administration, 2011.

H. Min, Y. Yang, Y. Fang, P. Sun, and X. Zhao. Constrained optimization and distributed model predictive control-based merging strategies for adjacent connected autonomous vehicle platoons. IEEE Access, 7: 163085-163096, 2019.

D. S. Naidu. Optimal control systems. CRC press, 2002.

I. A. Ntousakis, I. K. Nikolos, and M. Papageorgiou. Optimal vehicle trajectory planning in the context of cooperative merging on highways. Transportation Research Part C: Emerging Technologies, 71:464-488, 2016.

E. Ozatay, U. Ozguner, and D. Filev. Velocity profile optimization of on road vehicles: Pontryagin's maximum principle based approach. Control Engineering Practice, 61:244-254, 2017.

H. Pei, S. Feng, Y. Zhang, and D. Yao. A cooperative driving strategy for merging at on-ramps based on dynamic programming. IEEE Transactions on Vehicular Technology, 68(12):11646-11656, 2019.

X. Qian, J. Gregoire, A. De La Fortelle, and F. Moutarde.
Decentralized model predictive control for smooth coordination of automated vehicles at intersection. In 2015 European Control Conference (ECC), pages 3452-3458. IEEE, 2015.

G. Raravi, V. Shingde, K. Ramamritham, and J. Bharadia. Merge algorithms for intelligent vehicles. In Next Generation Design and Verification Methodologies for Distributed Embedded Control Systems, pages 51-65. 2007.

J. Rios-Torres and A. A. Malikopoulos. A Survey on Coordination of Connected and Automated Vehicles at Intersections and Merging at Highway On-Ramps. IEEE Transactions on Intelligent Transportation Systems, 18(5):1066-1077, 2017.

A. Sciarretta, G. De Nunzio, and L. L. Ojeda. Optimal ecodriving control: Energy-efficient driving of road vehicles as an optimal control problem. IEEE Control Systems Magazine, 35(5):71-90, 2015.

S. E. Shladover, C. A. Desoer, J. K. Hedrick, M. Tomizuka, J. Walrand, W.-B. Zhang, D. H. McMahon, H. Peng, S. Sheikholeslam, and N. McKeown. Automated vehicle control developments in the PATH program. IEEE Transactions on Vehicular Technology, 40(1):114-130, 1991.

P. Varaiya. Smart cars on smart roads: problems of control. IEEE Transactions on Automatic Control, 38(2): 195-207, 1993.

N. Wan, A. Vahidi, and A. Luckow. Optimal speed advisory for connected vehicles in arterial roads and the impact on mixed traffic. Transportation Research Part C: Emerging Technologies, 69:548-563, 2016.

J. Wang, X. Zhao, and G. Yin. Multi-objective optimal cooperative driving for connected and automated vehicles at non-signalised intersection. IET Intelligent Transport Systems, 13(1):79-89, 2019. ISSN 17519578. doi: 10.1049/iet-its.2018.5100.

B. Xu, X. J. Ban, Y. Bian, W. Li, J. Wang, S. E. Li, and K. Li. Cooperative method of traffic signal optimization and speed control of connected vehicles at isolated intersections. IEEE Transactions on Intelligent Transportation Systems, 20(4):1390-1403, 2018.

F. Yan, M. Dridi, and A. El Moudni. Autonomous vehicle sequencing algorithm at isolated intersections. 2009 12th International IEEE Conference on Intelligent Transportation Systems, pages 1-6, 2009.

Y. Zhang and C. G. Cassandras. Decentralized optimal control of connected automated vehicles at signal-free intersections including comfort-constrained turns and safety guarantees. Automatica, 109:108563, 2019.

L. Zhao, A. M. I. Mahbub, and A. A. Malikopoulos. Optimal vehicle dynamics and powertrain control for connected and automated vehicles. Proceedings of 2019 IEEE Conference on Control Technology and Applications (CCTA), pages 33-38, 2019. 